\title{
Modeling the resonant planetary system GJ 876
}

\author{
W. Kley ${ }^{1}$, M. H. Lee ${ }^{2}$, N. Murray ${ }^{3}$, and S. J. Peale ${ }^{2}$ \\ ${ }^{1}$ Institut für Astronomie \& Astrophysik, Abt. Computational Physics, Universität Tübingen, Auf der Morgenstelle 10, \\ 72076 Tübingen, Germany \\ e-mail: kley@tat.physik.uni-tuebingen.de \\ 2 Department of Physics, University of California, Santa Barbara, CA 93106, USA \\ ${ }^{3}$ Canada Research Chair in Astrophysics; CITA, University of Toronto, 60 St. George Street Toronto, Ontario M5S 3H8, \\ Canada
}

Received 7 January 2005 / Accepted 24 March 2005

\begin{abstract}
The two planets about the star GJ 876 appear to have undergone extensive migration from their point of origin in the protoplanetary disk - both because of their close proximity to the star (30 and 60 day orbital periods) and because of their occupying three stable orbital resonances at the 2:1 mean-motion commensurability. The resonances were most likely established by converging differential migration of the planets leading to capture into the resonances. A problem with this scenario is that continued migration of the system while it is trapped in the resonances leads to orbital eccentricities that rapidly exceed the observational upper limits of $e_{1} \approx 0.31$ and $e_{2} \approx 0.05$. As seen in forced 3-body simulations, these lower eccentricities would persist during migration only for an eccentricity damping rate $\dot{e}_{2} / e_{2}$ exceeding $\approx 40 \dot{a}_{2} / a_{2}$. Previous theoretical and numerical analyses have found $\dot{e} / e \sim \dot{a} / a$ or even eccentricity growth through disk-planet interactions.

In an attempt to find effects that could relax the excessive eccentricity damping requirement, we explore the evolution of the GJ 876 system using two-dimensional hydrodynamical simulations that include viscous heating and radiative cooling in some cases. Before we evolve the whole system, the disk with just the outer planet embedded is brought into equilibrium. We find that the relaxed disk remains circular in all models for low planet-to-star mass ratios $q_{2}$, but becomes eccentric for high mass ratios for those models with fixed temperature structure. The disk in models with full radiative thermodynamics remains circular for all $q_{2}$ considered due to the larger disk temperatures. Given the small stellar mass, the mass ratio for the GJ 876 system is rather high (with minimum $q_{2}=5.65 \times 10^{-3}$ ), and so the GJ 876 disk may have been slightly eccentric during the migration.

With a range of parameter values, we find that a hydrodynamic evolution within the resonance, where only the outer planet interacts with the disk, always rapidly leads to large values of eccentricities that exceed those observed - similar to the threebody results. The resonance corresponding to the resonant angle $\theta_{1}=2 \lambda_{2}-\lambda_{1}-\varpi_{1}$ (involving the inner planet's periapse longitude, $\varpi_{1}$ ) is always captured first. There is no additional delay in capturing $\theta_{2}=2 \lambda_{2}-\lambda_{1}-\varpi_{2}$ into resonance that is attributable to the secular prograde contribution to the precession of $\varpi_{2}$ from the interaction with the disk, but an eccentric disk can induce a large outer planet eccentricity $e_{2}$ before capture and thereby further delay capture of $\theta_{2}$ for larger planetary masses. The delay in capturing $\theta_{2}$ into libration, while delaying the resonance-induced growth of $e_{2}$, has no effect on the forced eccentricities of both planets, which are uniquely determined by the resonance conditions, once both $\theta_{j}$ are librating.

Only if mass is removed from the disk on a time scale of the order of the migration time scale (before there has been extensive migration after capture), as might occur for photoevaporation in the late phases of planet formation, can we end up with eccentricities that are consistent with the observations.
\end{abstract}

Key words. accretion, accretion disks - planets and satellites: formation - hydrodynamics - methods: N-body simulations

\section{Introduction}

Among the 14 known extrasolar planetary systems with multiple planets, at least three exhibit pairs of planets that are likely to be in orbital resonances at low order commensurabilities of their mean motions. The pair of planets around GJ 876 (Marcy et al. 2001) is well confirmed to be deep in the resonances associated with the 2:1 mean motion commensurability (Laughlin \& Chambers 2001; Rivera \& Lissauer 2001; Laughlin et al. 2004), where resonant angles $\theta_{1}=2 \lambda_{2}-\lambda_{1}-\varpi_{1}$, $\theta_{2}=2 \lambda_{2}-\lambda_{1}-\varpi_{2}$, and $\Delta \varpi=\varpi_{2}-\varpi_{1}=\theta_{1}-\theta_{2}$ are all librating about $0^{\circ}$ with small amplitudes. Here $\lambda_{j}$ are mean longitudes and $\varpi_{j}$ are longitudes of periapse, both numbered from the inside out. The pair of planets around HD 82943 is likely to also be in resonances at 2:1 (Mayor et al. 2004), and the middle pair of planets in the 4 planet system orbiting $55 \mathrm{Cnc}$ may be in resonances at 3:1 (Marcy et al. 2002; McArthur et al. 2004). Thus as many as one-fourth of multiple-planet systems contain planets in mean motion resonances.

Both theoretical and numerical analyses verify the ubiquity of planet migration due to interaction with the protoplanetary 
disk of gas and dust (e.g. Ward 1997; Nelson et al. 2000). The clearing of disk material between two planets both capable of opening a gap in the disk leads to differential migration of the two planets as at least the outer planet is forced in by the material remaining outside its orbit. The convergence of the orbits naturally allows capture into stable resonant orbital configurations. Hydrodynamical simulations of two embedded planets capturing each other into resonance have been performed by several groups (Kley 2000; Bryden et al. 2000; Snellgrove et al. 2001; Papaloizou 2003; Kley et al. 2004). The resonance capture has also been analyzed with extensive three-body calculations, where migration is simply imposed with either the semimajor axis migration rate $\dot{a} / a$ and the eccentricity damping rate $\dot{e} / e$ specified explicitly or ad hoc forces added to produce migration and eccentricity damping (Snellgrove et al. 2001; Lee \& Peale 2002; Nelson \& Papaloizou 2002; Kley et al. 2004; Lee 2004).

Depending on the migration rates, masses, and initial orbital separations and eccentricities of the two planets, capture can occur in different resonances. For planets as massive as those in GJ 876, capture into the 2:1 resonances is robust if the initial $a_{2} / a_{1} \lesssim 2$ and the initial eccentricities are small, and it is most likely that the 2:1 resonances for GJ 876 were established by converging differential migration (Lee \& Peale 2002). The sequence of 2:1 resonance configurations that a system with initially nearly circular orbits is driven through by continued migration depends mainly on the planetary mass ratio $M_{1} / M_{2}$. For $M_{1} / M_{2} \approx 0.31$ as in the GJ 876 system, a system is first captured into antisymmetric configurations with $\theta_{1}$ librating about $0^{\circ}$ and $\Delta \varpi$ (and hence $\theta_{2}$ ) librating about $180^{\circ}$. Continued migration forces $e_{1}$ to larger values and $e_{2}$ from increasing to decreasing until $e_{2} \approx 0$ when $e_{1} \approx 0.1$ Then the system converts to symmetric configurations like that of GJ 876 , with both $\theta_{1}$ and $\Delta \varpi$ librating about $0^{\circ}$ (Lee 2004; see also Fig. 16 below). There are other types of 2:1 resonance configurations (with both $\theta_{1}$ and $\Delta \varpi$ librating about $180^{\circ}$ or asymmetric librations of $\theta_{1}$ and $\Delta \varpi$ about values far from either $0^{\circ}$ or $180^{\circ}$ ) for $M_{1} / M_{2} \approx 0.31$, but they are either unstable for planets as massive as those in GJ 876 or not reachable by convergent migration of planets with nearly constant masses and coplanar orbits (Lee 2004). Asymmetric libration configurations can result from convergent migration for 2:1 resonances with $M_{1} / M_{2} \gtrsim 0.95$ and for 3:1 resonances for a wider range of $M_{1} / M_{2}$ (Beaugé et al. 2003; Ferraz-Mello et al. 2003; Kley et al. 2004; Lee 2004).

While it is easy to understand the symmetric resonance configuration of GJ 876 from convergent migration, the small eccentricities of this system are a puzzle. The continued migration after capture into resonance drives the pair of planets deeper into the resonances $\left(2 n_{2}-n_{1}<0\right.$ increasing, with $n_{j}=\dot{\lambda}_{j}$ being the mean orbital motions). The identical mean retrograde precessions of the $\varpi_{j}$ must therefore decrease in magnitude to keep $\dot{\theta}_{j}$ near zero, i.e., to maintain the resonance configuration. This decrease is effected by increasing the orbital eccentricities when the system is in the configuration with both $\theta_{1}$ and $\theta_{2}$ librating about $0^{\circ}$. The three-body calculations have shown that the eccentricity growth is rapid if there is no eccentricity damping, with the average eccentricities $\left(\left\langle e_{1}\right\rangle=0.255,\left\langle e_{2}\right\rangle=0.035\right)$ of the $\sin i=0.78$ fit of Laughlin \& Chambers (2001) exceeded after only a $7 \%$ decrease in the orbital radii after capture (Lee \& Peale 2002). The eccentricities can be maintained at the observed low values as migration continues if there is significant eccentricity damping of $\dot{e} / e \approx 100 \dot{a} / a$ (Lee \& Peale 2002). In contrast the full hydrodynamical calculations typically give similar timescales for both migration and eccentricity damping (Kley et al. 2004). The low eccentricities become even more problematic if disk-planet interactions drive eccentricity growth. Ogilvie \& Lubow (2003) and Goldreich \& Sari (2003) have suggested that the eccentricity-damping corotation torques can be reduced sufficiently to trigger eccentricity growth if the eccentricity is above a critical value, and the eccentricity of the outer planet in GJ 876 is well above their estimates for this critical value.

Hydrodynamical simulations of two planets interacting with their protoplanetary disk are computationally expensive, and the number of such simulations in the literature is relatively small (Kley 2000; Bryden et al. 2000; Snellgrove et al. 2001; Papaloizou 2003; Kley et al. 2004). In addition, often the masses used in these simulations are not appropriate for the GJ 876 system or the simulations did not continue for a very long time. While there has been more extensive three-body calculations with imposed migration and eccentricity damping (Snellgrove et al. 2001; Lee \& Peale 2002; Nelson \& Papaloizou 2002; Kley et al. 2004; Lee 2004), they do not model disk-planet interactions self-consistently and, in particular, the effects of the apsidal precession induced by the disk have not been considered.

In this paper we present a more comprehensive set of numerical calculations treating the evolution of two planets interacting with their protoplanetary disk, with special emphasis on the resonant system GJ 876. For this purpose we solve the full hydrodynamical equations, including viscous heating and radiative cooling effects in some cases, and follow the joint planet-disk evolution over several thousand orbits of the planets. We find that we cannot reproduce the observed low eccentricities using these straightforward assumptions. Taking into account mass loss from the disk, as might be the case in the late dissipation stages of protoplanetary disks subject to, e.g., photoevaporation, we are able to reduce the final values of the eccentricities close to the observed values.

In Sect. 2 we give an overview of the observational data for GJ 876. In Sect. 3 we explain our physical and numerical approach, and in Sect. 4 we present the results of our simulations. This is followed in Sect. 5 with three-body calculations and analytic theory to interpret the results of Sect. 4. We state our conclusions in Sect. 6.

\section{The system GJ 876}

The first planet orbiting GJ 876 was discovered in 1998 (Marcy et al. 1998; Delfosse et al. 1998). With the discovery of the second inner planet (Marcy et al. 2001), the near 2:1 commensurability of the orbital periods ( $\approx 30$ and 60 days) implied stable mean-motion resonances, which were soon confirmed. The combined minimum mass of the planets is about 
Table 1. Orbital parameters of the two planets of the planetary system GJ 876 at epoch JD 2449679.6316 assuming co-planarity and $i=90^{\circ}$ as given by Laughlin et al. (2004). The adopted stellar mass is $M_{*}=$ $0.32 M_{\odot} . P$ denotes the orbital period, $M$ the mass of the planet, $a$ the semi-major axis, $e$ the eccentricity, $\varpi$ the angle of periastron at epoch, and $q$ the mass ratio $M / M_{*}$.

\begin{tabular}{lllllll}
\hline \hline & $P$ & $M$ & $a$ & $e$ & $\varpi$ & $q$ \\
& {$[\mathrm{~d}]$} & {$\left[M_{\text {Jup }}\right]$} & {$[\mathrm{AU}]$} & & {$[\mathrm{deg}]$} & {$\left[10^{-3}\right]$} \\
\hline Inner & 30.38 & 0.597 & 0.13 & 0.218 & 154 & 1.78 \\
Outer & 60.93 & 1.89 & 0.21 & 0.029 & 149 & 5.65 \\
\hline
\end{tabular}

0.0074 of the stellar mass $\left(M_{*}=0.32 M_{\odot}\right)$. The large relative planetary masses and short orbital periods meant that a dynamical fit to the radial velocity data that accounted for the mutual gravitational interaction of the planets was required. This was accomplished by Laughlin \& Chambers (2001) and Rivera \& Lissauer (2001), who used a Levenberg-Marquardt minimization scheme driving an $\mathrm{N}$-body integrator to find best-fit initial orbital elements. Recently new data obtained with the Keck Telescope have also been included in the analysis of the system (Laughlin et al. 2004), and the latest best-fit orbital parameters are shown in Table 1 for an assumed coplanar orbital inclination of $i=90^{\circ}$.

Although a dynamical fit can in principle yield the orbital inclinations and masses of the planets, the $\chi^{2}$ value of the fit for GJ 876 is relatively insensitive to the inclination $i$ of the assumed coplanar orbits for $35^{\circ} \leqslant i \leq 90^{\circ}$ and rises rapidly only for $i \lesssim 35^{\circ}$ (Laughlin et al. 2004). In Fig. 1 we show the planetary mass ratio $M_{1} / M_{2}$ and the average orbital eccentricities $\left\langle e_{1}\right\rangle$ and $\left\langle e_{2}\right\rangle$ for the best-fits found by Laughlin et al. (2004) for $i>35^{\circ}$. The deviation from Keplerian motion that is most strongly constrained by the available observations of GJ 876 is the resonance induced retrograde apsidal precession of the orbits at an average rate of $\dot{\varpi}=-41^{\circ} \mathrm{yr}^{-1}$, and indeed this precession has now been observed for more than one full period. As $i$ decreases, the individual planetary mass $M_{j}$ increases roughly as $1 / \sin i$ (and $M_{1} / M_{2}$ is nearly constant and $\approx 0.31$ ). The increase in planetary mass acts to increase $\dot{\varpi}$. This increase can be offset by an increase in $\left\langle e_{j}\right\rangle$, which acts to decrease $\dot{\varpi}$, keeping $\dot{\varpi}$ near the observed value without any significant change in $\chi^{2}$ until $i \lesssim 35^{\circ}$. To allow for the mass uncertainties, we shall consider systems with different planetary masses in our numerical models.

The most striking feature of GJ 876 is the exact 2:1 orbital resonance of the planets. The angles $\theta_{1}, \theta_{2}$, and $\Delta \varpi$ describe the state of the system for coplanar orbits, and all three angles librate about $0^{\circ}$ in the GJ 876 system. The libration amplitudes are $\left|\theta_{1}\right|_{\max }=7^{\circ},\left|\theta_{2}\right|_{\max }=34^{\circ}$, and $|\Delta \varpi|_{\max }=34^{\circ}$ for the $i=90^{\circ}$ fit shown in Table 1 , and the smallest libration amplitudes in the range $35^{\circ} \leq i \leq 90^{\circ}$ are $\left|\theta_{1}\right|_{\max } \approx 5^{\circ}$ at $i \approx 50^{\circ}$ and $\left|\theta_{2}\right|_{\max } \approx 16^{\circ}$ and $|\Delta \varpi|_{\max } \approx 13^{\circ}$ at $i \approx 35^{\circ}$ (Laughlin et al. 2004). The small libration amplitudes indicate that the system is deep in the resonance, which occurs naturally in the differential migration scheme for forming the resonant structure if the planets approach the resonance slowly with initially small eccentricities (Lee \& Peale 2002).

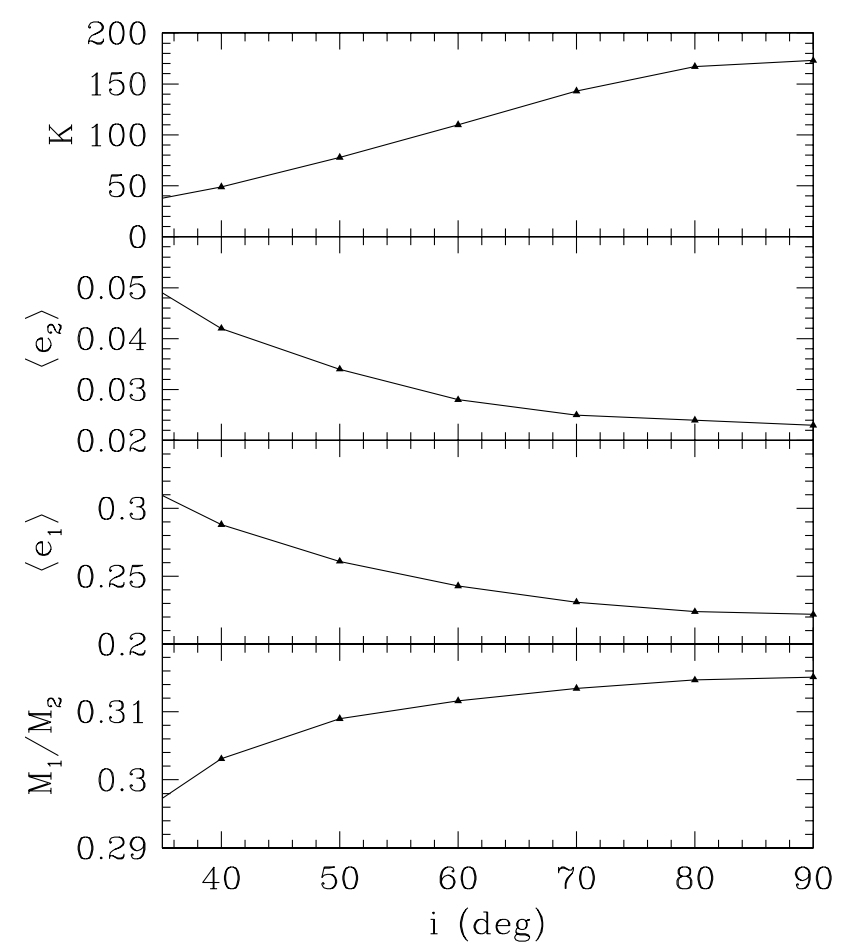

Fig. 1. Planetary mass ratio, $M_{1} / M_{2}$, average orbital eccentricities, $\left\langle e_{1}\right\rangle$ and $\left\langle e_{2}\right\rangle$, and the ratio of eccentricity damping rate to migration rate, $K$, for the GJ 876 best-fit solutions of Laughlin et al. (2004) with coplanar inclination $i>35^{\circ}$. The value of $K=\left|\dot{e}_{2} / e_{2}\right| /\left|\dot{a}_{2} / a_{2}\right|$ is for the equilibrium eccentricities to match $\left\langle e_{j}\right\rangle$ if there is inward migration and damping of the outer planet only.

Lee \& Peale (2002) have shown using three-body integrations with imposed migration and eccentricity damping that the eccentricities reach equilibrium values that remain constant for arbitrarily long migration within the resonances if $\dot{e}_{j} / e_{j}=-K\left|\dot{a}_{j} / a_{j}\right|$. For inward migration and damping of the outer planet only, $K \approx 100$ is required to produce equilibrium eccentricities that match the eccentricities of the $\sin i=0.78$ (or $i=51^{\circ}$ ) fit of Laughlin \& Chambers (2001). We have repeated the calculations in Lee \& Peale (2002) for the updated fits in Laughlin et al. (2004), and the value of $K$ as a function of $i$ is shown in Fig. 1 . For $i=51^{\circ}, K \approx 80$, which is close to the value inferred by Lee \& Peale (2002). Benedict et al. (2002) have claimed from HST astrometric measurements that the inclination is very close to $90^{\circ}$, which would require a significantly larger amount of eccentricity damping $(K \approx 170)$. The increase in $\left\langle e_{j}\right\rangle$ with decreasing $i$ leads to a decrease in $K$, but $K$ must still be greater than about 40 if $i \gtrsim 35^{\circ}$.

\section{The hydrodynamical model}

Even though the physical setup is different from existing simulations, the models presented here are calculated roughly in the same manner as those described previously in Kley (1998, 1999) for single planets and in Kley (2000) for multiple planets. The reader is referred to those papers for details on the computational aspects of this type of simulations. Other similar models, following explicitly the motion of single and multiple planets in disks, have been presented by Nelson et al. (2000), 
Bryden et al. (2000), Snellgrove et al. (2001), Papaloizou (2003), and more recently for resonant configurations by Kley et al. (2004).

We use cylindrical coordinates $(r, \varphi, z)$ and consider a vertically averaged, infinitesimally thin disk located at $z=0$, where the origin of the coordinate system is at the position of the star. The basic hydrodynamic equations (mass and momentum conservation) describing the time evolution of such a twodimensional disk with embedded planets have been stated frequently and are not repeated here (see Kley 1999). Additional information on the treatment of embedded planets is given in Kley et al. (2004).

\subsection{Energy equation}

The majority of models presented here use a fixed temperature distribution which follows from the assumption of a constant ratio of vertical height $H(r)$ to radial distance $r$ from the star. Here we assume $H(r) / r=$ const. $=0.05$, from which $T(r) \propto r^{-1}$ follows. In this case the pressure $p$ is given by $p=\Sigma c_{\mathrm{s}}^{2}$, where $\Sigma$ is the surface density and $c_{\mathrm{s}}$ the isothermal sound speed. In the situation of a given $H / r$-ratio there is no need for solving an extra energy equation, and we refer to those models as isothermal (even though the radial temperature is varying).

We also present radiative models with an improved thermodynamic treatment using the thermal energy equation

$\frac{\partial \Sigma c_{\mathrm{v}} T}{\partial t}+\nabla \cdot\left(\Sigma c_{\mathrm{v}} T \mathbf{u}\right)=-p \nabla \cdot \mathbf{u}+D-Q$.

Here, $\boldsymbol{u}=\left(u_{r}, u_{\varphi}\right)$ is the two-dimensional velocity, $T$ the (midplane) temperature of the disk, $c_{\mathrm{v}}$ the ratio of specific heats, $D$ the dissipation function, and $Q$ is the local radiative cooling. The form of the dissipation function $D$ in cylindrical coordinates can be found in Mihalas \& Weibel Mihalas (1984), and to calculate the radiative losses (from the two sides of the disk) we follow D'Angelo et al. (2003a) and Günther et al. (2004) and write

$Q=2 \sigma_{\mathrm{R}} T_{\mathrm{eff}}^{4}$

where $\sigma_{\mathrm{R}}$ is the Stefan-Boltzmann constant and $T_{\text {eff }}$ is an estimate for the effective temperature (Hubeny 1990)

$T_{\text {eff }}^{4} \tau_{\text {eff }}=T^{4} \quad$ with $\quad \tau_{\text {eff }}=\frac{3}{8} \tau+\frac{\sqrt{3}}{4}+\frac{1}{4 \tau}$.

For a two-dimensional disk we approximate the mean vertical optical depth by

$\tau=\frac{1}{2} \kappa \Sigma$

where for the Rosseland mean opacity $\kappa$ the analytical formulae by Lin \& Papaloizou (1985) are adopted.

In addition to the simplified treatment of radiation as expressed in Eq. (1) we also have considered models including radiative diffusion in the $(r, \varphi)$-plane. In this case a term

$-2 H \nabla \cdot \boldsymbol{F} \quad$ with $\quad \boldsymbol{F}=-\frac{\lambda c 4 a T^{3}}{\rho \kappa} \nabla T$

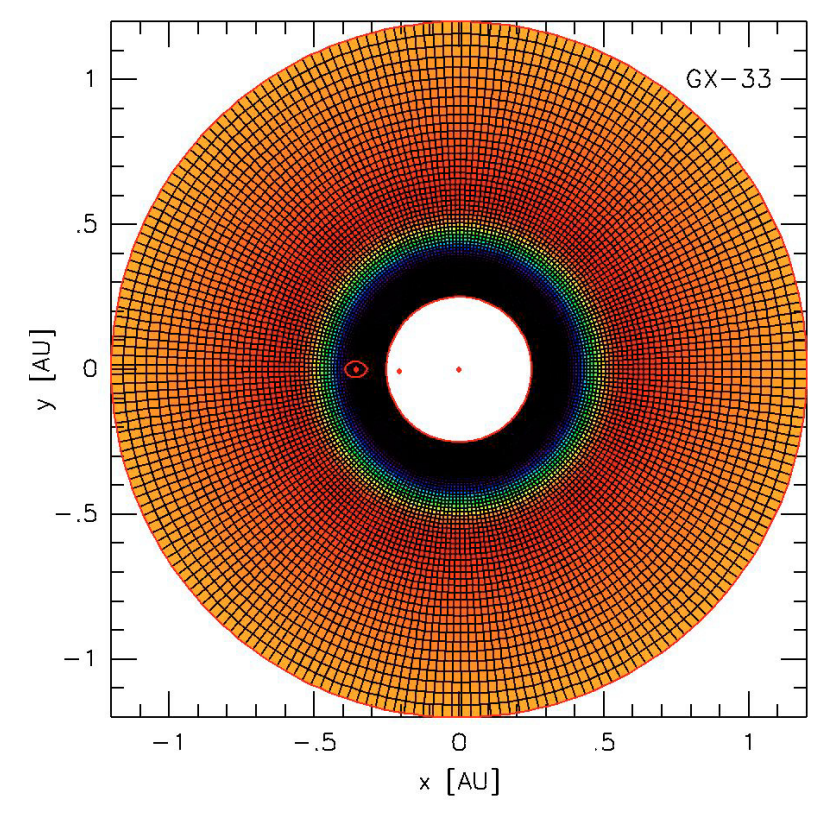

Fig. 2. The global grid structure with gray-shaded initial surface density superimposed. Every second grid points is shown. The dots denote the location of the star and the two planets and the oval line refers to the Roche lobe of the outer planet.

has been added to the rhs of Eq. (1). Here $\boldsymbol{F}$ denotes the radiative flux in the $(r, \varphi)$-plane, $c$ is the speed of light, $a$ the radiation constant, $\rho=\Sigma /(2 H)$ the midplane density, and $\lambda$ the flux-limiter (see Kley 1989).

We work in a rotating reference system, rotating approximately with the initial period of the outer planet. As the coordinate system is accelerated and rotating, we take care to include the indirect terms.

\subsection{Initial setup}

The two-dimensional $(r-\varphi)$ computational domain consists of a complete ring of the protoplanetary disk centered on the star. By previous simulations of two embedded planets interacting with the protoplanetary disk it has been shown that the inner part of the disk (inside of the outer planet) clears rapidly. The final configuration is such that two planets orbit the star inside a cavity (Kley et al. 2004). In this state only the outer planet is still in touch with the disk, see Fig. 1 in Kley et al. (2004). Taking account of this fact, we simplify the setup and choose the radial extent of the computational domain (ranging from $r_{\min }$ to $r_{\max }$ ) such that the inner planet orbits entirely inside $r_{\min }$. This reduces the necessary radial range covered and leads to a significant reduction in computational effort. A typical example of the grid structure is displayed in Fig. 2. In the azimuthal direction for a complete annulus we have $\varphi_{\min }=0$ to $\varphi_{\max }=$ $2 \pi$.

The initial hydrodynamic structure of the disk (density, temperature, velocity) is axisymmetric with respect to the location of the star, and the surface density scales as $\Sigma(r)=\Sigma_{0} r^{-1 / 2}$, with a superimposed initial gap (Kley 2000). The initial velocity is pure Keplerian rotation $\left(u_{r}=0, u_{\varphi}=\left(G M_{*} / r\right)^{1 / 2}\right)$, and the initial temperature stratification is given by $T(r) \propto r^{-1}$ 
which follows from an assumed constant $H / r$. For the isothermal models the initial temperature profile is left unchanged, while for the radiative cases it is evolved according to Eq. (1).

The kinematic viscosity $v$ is parameterized by an $\alpha$ description $v=\alpha c_{\mathrm{s}} H$, where the isothermal sound-speed is given by $c_{\mathrm{s}}=\left(c_{\mathrm{v}} T\right)^{1 / 2}$, and $H(r)$ is either held fixed (for the isothermal models) or, for the radiative models, calculated from the temperature/sound-speed as $H(r)=c_{\mathrm{s}} / \Omega_{\mathrm{K}}(r)$, where

$\Omega_{\mathrm{K}}=\left(\frac{G M_{*}}{r^{3}}\right)^{1 / 2}$

is the Keplerian angular velocity of the disk.

\subsection{Boundary conditions}

In an effort to ensure a uniform environment for all models and minimize disturbances (wave reflections) from the outer boundary, we impose at $r_{\max }$ damping boundary conditions where the density and both velocity components are relaxed towards their initial state as

$\frac{\mathrm{d} X}{\mathrm{~d} t}=-\frac{X-X(t=0)}{\tau_{\mathrm{damp}}} R(r)^{2}$

where $X \in\left\{\Sigma, u_{r}, u_{\varphi}\right\}, \tau_{\mathrm{damp}}=1 / \Omega_{\mathrm{K}}\left(r_{\max }\right)$ and $R(r)$ is a dimensionless linear ramp-function rising from 0 to 1 within $\left[r_{\mathrm{damp}}, r_{\max }\right]$. This damping setup is defined in more detail in an international comparison test project ${ }^{1}$. As the initial radial velocity is vanishing, this damping routine ensures that no mass flows through the outer boundary at $r_{\max }$. However, in some models described below, matter is allowed to leave the outer boundary.

At the inner radial boundary $r_{\text {min }}$ outflow conditions are applied; matter may flow out, but none is allowed to enter. This procedure mimics the accretion process onto the star. The density gradient is set to zero at $r_{\text {min }}$, while the angular velocity there is fixed to be Keplerian. In the azimuthal direction, periodic boundary conditions for all variables are imposed.

These specified boundary conditions allow for a well defined quasi-stationary state if the planets are not allowed to respond to the disk.

\subsection{Model parameters}

The computational grid in all models has the same radial extent from $r_{\min }=0.25$ to $r_{\max }=1.20 \mathrm{AU}$. It is covered by $111 \times$ $450\left(N_{r} \times N_{\varphi}\right)$ gridcells which are spaced logarithmically in radius and equidistant in azimuth. The radius beyond which the damping procedure defined above gradually sets in is given by $r_{\text {damp }}=1.1$. The stellar mass is $0.32 M_{\odot}$ in all models. For the planetary masses different choices have been made as the exact parameters for GJ 876 are not known. The majority of models assumed an edge on system $i=90^{\circ}$ which leads to (see Table 1) $m_{1}=0.597 M_{\text {Jup }}$ and $m_{2}=1.89 M_{\text {Jup }}$. Recall that the index 1 refers to the inner and the index 2 to the outer planet. In terms of their mass ratios $\left(M / M_{*}\right)$ these values are

\footnotetext{
${ }^{1}$ http://www.astro.su.se/ pawel/planets/ test.hydro.html
}

equivalent to $q_{1}=1.78 \times 10^{-3}$ and $q_{2}=5.65 \times 10^{-3}$. In the analysis of the models we prefer to state the planetary masses in terms of mass ratios rather than the actual values. Most of the mass ratios used in this investigation are close to the edgeon case. The two planets are placed initially at semi-major axes of $a_{1}=0.20 \mathrm{AU}$ and $a_{2}=0.35 \mathrm{AU}$. Both planets have an initial eccentricity of 0.01 which is comparable to what is found typically in numerical simulations of disk-planet interactions. Even if the planets were started with zero initial eccentricity at those $a_{j}$, the dynamical interaction between the planets alone would also lead to non-zero eccentricities of the same order.

After an initial relaxation procedure, where the orbital parameters of the planets are not changed and the disk structure is brought to an equilibrium, the planets are "released" in all cases and are allowed to migrate (change their semi-major axes) in accordance with the gravitational disk forces/torques exerted on them.

During the evolution, material may enter from the disk into the Roche lobe of a planet. This material is partly removed from the simulation to model accretion onto the planet; for the detailed procedure see Kley (1999). In some models this mass is not added to the dynamical mass of the planets, so that mass is not strictly conserved. In other simulations the mass is added to that of the planet, maintaining conservation of mass. In Table 2 below it is indicated that only one of the presented models (h8a) has a varying dynamical mass. In general it does not change the outcome of a simulation noticably whether mass is accreted onto a planet or not.

For the viscosity a value of $\alpha=0.01$ is used for all models. This is probably on the large side for protoplanetary disks, but it allows for a rapid evolution of the system and hence a reasonable computational effort; a larger $\alpha$ speeds up the evolution and migration of the planet. It has been shown earlier that the migration speed has no influence on final magnitude of the eccentricities (Lee \& Peale 2002) which tends to justify this approach. In addition, a larger $\alpha$-value leads to a gap that is not so well cleared (Kley 1999) which will tend to induce a larger periastron advance $(\dot{\varpi})$ and an increased eccentricity damping. Both effects presumably serve to minimize the final eccentricity of the outer planet.

The density in the system is adjusted such that in the relaxed initial state there is approximately $2.75 \times 10^{-3} M_{\odot}$ of material within the computational domain (see below).

\subsection{A few remarks on numerical issues}

The numerical method used is a staggered mesh, spatially second order finite difference method, where advection is based on the monotonic transport algorithm (van Leer 1977). The code uses operator-splitting and is semi-second order in time. The computational details of the code that we employ, RH2D, have been described in general in Kley (1989), and specifically for planet calculations in Kley (1999). The use of a rotating coordinate system requires special treatment of the Coriolis terms to ensure angular momentum conservation (Kley 1998), an especially important point for the long-term calculations presented here. 
The viscous terms, including all necessary tensor components, are treated explicitly. To ensure stability in the gap region, where there are very strong gradients in the density, an artificial bulk viscosity has been added, with a coefficient $C_{\text {art }}=2$. For a detailed discussion of the viscosity related issues and tests, see Kley (1999).

The energy equation Eq. (1) is solved explicitly applying operator-splitting. The heating and cooling term $D-Q$ is treated as one sub-step in this procedure, see Günther et al. (2004). The additional radiative diffusion part in the energy Eq. (5) is solved applying an implicit method to avoid possible time step limitations. To solve the resulting matrix equations we use Successive Over Relaxation (SOR) with an adaptive relaxation parameter (Kley 1989).

A larger mass ratio $M / M_{*}$ induces stronger torques and produces low densities in the gap region. To prevent numerical instabilitites caused by too large gradients we have found it preferable to work with a density floor, where the density cannot fall below a specified minimum value $\Sigma_{\min }$. For our purpose we use a value of $\Sigma_{\min }=10^{-6}$ in dimensionless values, where the typical (initial) density is of $O(1)$.

\subsubsection{Planetary dynamics}

The motion of the planets is integrated using a fourth order Runge-Kutta integrator where the time step size is given by the hydrodynamical time step. While not the most accurate integrator for full $N$-body calculations, it is sufficient for our purposes. As a test we have run the pure 3-body problem of one star with two planets under exactly the same conditions as in the full hydrodynamical evolution and find that the relative total energy loss is less than $6 \times 10^{-6}$ in 1000 years $\left(\approx 2.5 \times 10^{6}\right.$ time steps $)$, which is equivalent to over 6000 periods of the inner and over around 2700 periods of the outer planet.

The forces of the disk are taken into account in a first order approximation to reduce the computational effort. To avoid problems with under-resolved material close to the planet, a torque cutoff radius of $r_{\text {torq }}$ is applied where material closer to the planet than $r_{\text {torq }}$ does not contribute to the force acting on the planet. The problem of choosing the optimum value for $r_{\text {torq }}$ is non-trivial. Using a cutoff radius prevents large unphysical variations of the forces acting on the planet. Very high resolution simulations (D'Angelo et al. 2002, 2004) show a nearly symmetric distribution of material close to the planet indicating that those regions do not contribute too much to the total torque. As these regions cannot be resolved in our simulations we have to use a torque cutoff instead. For all isothermal models we use $r_{\text {torq }}=0.5 R_{\text {Hill }}$, where the Hill radius is given by

$R_{\mathrm{Hill}}=a_{2}\left(\frac{q_{2}}{3}\right)^{1 / 3}$.

Tests with an increased value of $0.75 R_{\text {Hill }}$ gave indistinguishable results on the migration rate and eccentricity evolution. For the radiative models we use a value of $r_{\text {torq }}=0.8 R_{\text {Hill }}$ because the radiative cooling leads to more material around the planet.

In calculating the gravitational potential of the disk and planet the vertical extent of the disk is taken into account, assuming a vertically isothermal structure, i.e. Gaussian density distribution. For the smoothing length of the potential we choose $r_{\text {pot }}=0.8 H$. For a $1.9 M_{\text {Jup }}$ planet this is equivalent to only about $0.3 R_{\text {Hill }}$.

\section{Model computations}

Constructing our models for the formation of GJ 876 consists of two basic steps:

I. construct equilibrium models where the planets have fixed orbital elements, and let the disk evolve into a quasi-stationary state;

II. follow the subsequent evolution by 'releasing' the planets, i.e. by taking into account the disk forces acting on the planet. This two-fold procedure is necessary to avoid evolutions which might be dominated by a transient adjustment of the disk, as the disk-planet equilibrium state is not known a priori. The disadvantage is that the equilibration phase may take many hundreds of orbits. Let us consider these two steps in turn.

\subsection{Relaxation towards equilibrium}

\subsubsection{Switched off inner planet}

In the first part of step I the mass of the inner planet is switched off (reduced by a factor $10^{-6}$ ) and the outer planet has fixed orbital elements $\left(a_{2}=0.35, e_{2}=0.01\right)$. We construct equilibrium models for different planet masses $q_{2}$ because firstly, the observations do not yield definite masses of the planets due to the poorly constrained inclination, and secondly we want to understand the planet-disk system in more general terms. If the surface density and all other variables are fixed at the outer boundary there corresponds (for a given viscosity and vertical height of the disk) one particular equilibrium state to each mass ratio.

In Fig. 3 the azimuthally averaged $\Sigma(r)$ profile is shown for the relaxed equilibrium disk states for three different masses $q_{2}$ of the outer planet starting from $q_{2}=1.0 \times 10^{-3}$ (black solid line), over $3.5 \times 10^{-3}$ (gray dotted line) upto $5.9 \times 10^{-3}$ (dashed line), with the last value close to the minimum mass of the outer planet of the GJ 876 system. One notices that for the two smaller planetary masses the outer disk is much more "quiescent" than for the large mass. Larger planetary masses apparently lead to a restructuring of the disk. The wave-like disturbances in the disk (seen as the oscillatory behavior in the curves) are significantly stronger for $q_{2} \gtrsim 5.2 \times 10^{-3}$.

The existence of the two different equilibrium states of the disk is further illustrated in Fig. 4 where we display gray scale plots of the surface density $\Sigma$ for the relaxed state with no inner planet for two different masses $\left(q_{2}=3.5\right.$ and $\left.5.9 \times 10^{-3}\right)$. In both cases the small but non-vanishing eccentricity of the outer planet $\left(e_{2}=0.01\right)$ leads to wave-like disturbances in the disk oscillating with the planetary period. But while for the lower mass case $\left(q_{2}=3.5 \times 10^{-3}\right)$ the disk structure remains quite regular, the second high mass case $\left(q_{2}=5.9 \times 10^{-3}\right)$ shows a strongly disturbed disk which has gained a significant eccentricity of about 0.25 near the gap edge. The transition from the non-eccentric state to the eccentric state which is here a func- 


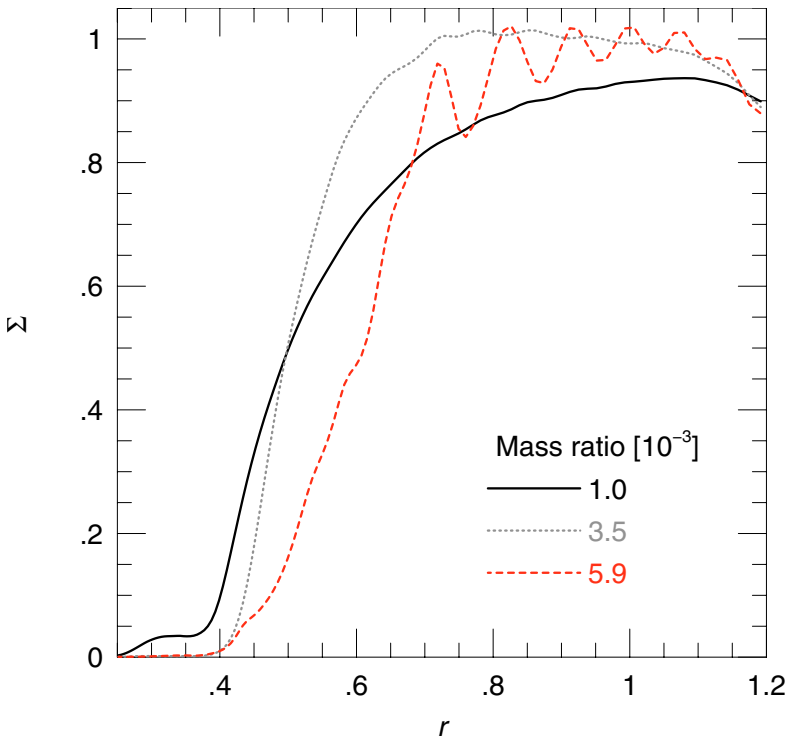

Fig. 3. The azimuthally averaged density profile for the relaxed configurations for three different masses of the outer planet: $q_{2}=1.0 \times$ $10^{-3}, q_{2}=3.5 \times 10^{-3}$ and $5.9 \times 10^{-3}$. The planet is located at $a_{2}=0.35$ with a fixed semi-major axis, and is allowed to accrete. In these models the mass of the inner planet has been switched off. The density is given in dimensionless units.

tion only of the planetary mass may depend also on the viscosity and temperature on the disk which we have held fixed. To check if this effect is induced by the non-zero eccentricity of the planet we have run a model with a vanishing eccentricity $\left(e_{2}=0\right)$ and found the same behavior. We find that the eccentric disk is nearly stationary in the inertial frame. The mass flow onto the planet and through the gap is higher than the flow for the non-eccentric disk that prevailed for the lower mass planet. While it would be interesting, a further exploration of these features is beyond the scope of this paper, and we leave it to a subsequent study. This disk eccentricity may be closely related to the disk eccentricity induced by resonant interaction of the outer 3:1 eccentric Lindblad resonance of planet 2 with the disk, as discussed by Papaloizou et al. (2001), and to the problem of the inner disk in cataclysmic variables by Lubow (1991). This violent interaction of a massive planet with the disk explains also some of the outlined numerical difficulties and requirements (such as density floor and artificial viscosity).

\subsubsection{Evolution of the outer planet alone}

To explore the effect the disk alone has on the evolution of the orbital elements of the single (outer) planet, in particular the influence on the precession rate, we present some calculations where the gravitational back-reaction of the disk is taken into account, while the inner planet is not present. For this purpose we chose the density unit such that $\Sigma=1$ (e.g. in Fig. 3) refers to a surface density of approximately $7500 \mathrm{~g} \mathrm{~cm}^{-2}$. This value refers to a total mass of $2.75 M_{\text {Jup }}$ within the computational domain for the $q_{2}=0.0059$ case.

In Figs. 5 and 6 the time evolution of the orbital elements of the outer planet for three models with different planet masses
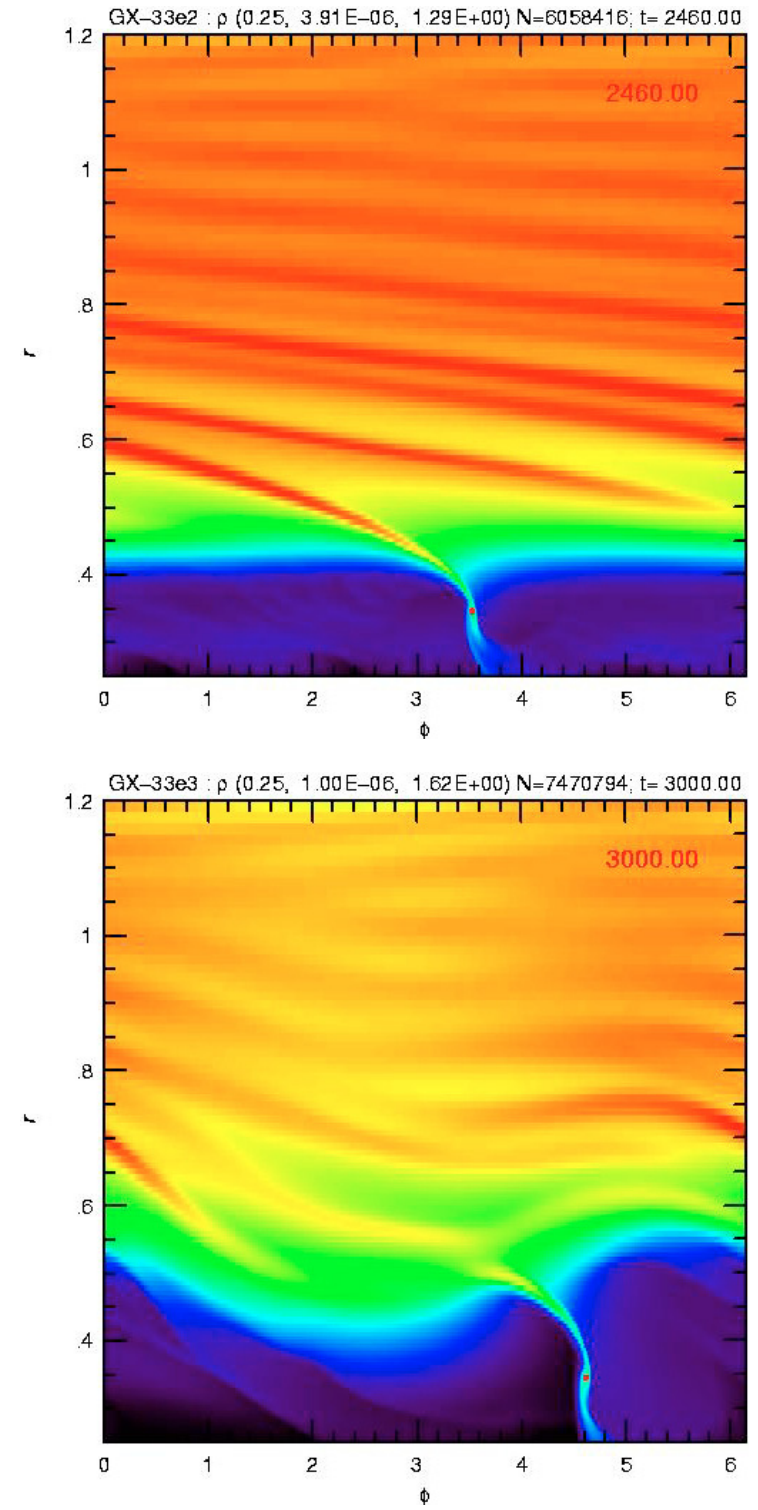

Fig. 4. Gray scale plots of the surface density $\Sigma$ for the relaxed state with no inner planet for two different masses: top: $q_{2}=3.5 \times 10^{-3}$ and bottom: $q_{2}=5.9 \times 10^{-3}$. Due to the higher planetary mass much stronger wave-like disturbances are created in the density, and the disk becomes eccentric with very small pattern speed in the inertial frame.

are displayed. All models have been relaxed before releasing the planet. For direct comparison the physical disk mass in all three cases has been scaled to be identical $\left(\approx 2.75 \times 10^{-3} M_{\odot}\right)$. With respect to the reference model (c3) with $q_{2}=0.0059$, this implies for models (d3a) and (e4a) a density reduction factor of 0.98 and 0.9 , respectively (cp. Fig. 3 ). The migration rate of the planets depends primarily on the amount of mass near the 2:1 Lindblad resonance. We observe that the lowest $\left(q_{2}=0.001\right)$ and highest $\left(q_{2}=0.0059\right)$ mass planet have very similar initial migration rates, while the intermediate mass planet has a faster migration rate. The intermediate mass $\left(q_{2}=3.5 \times 10^{-3}\right)$ has the largest rate because there is more mass very close to the location of the 2:1 resonance, which lies here at $r=0.56$ (note the steep density gradient in Fig. 3). From the average density profile one might have suspected a smaller migration rate for 

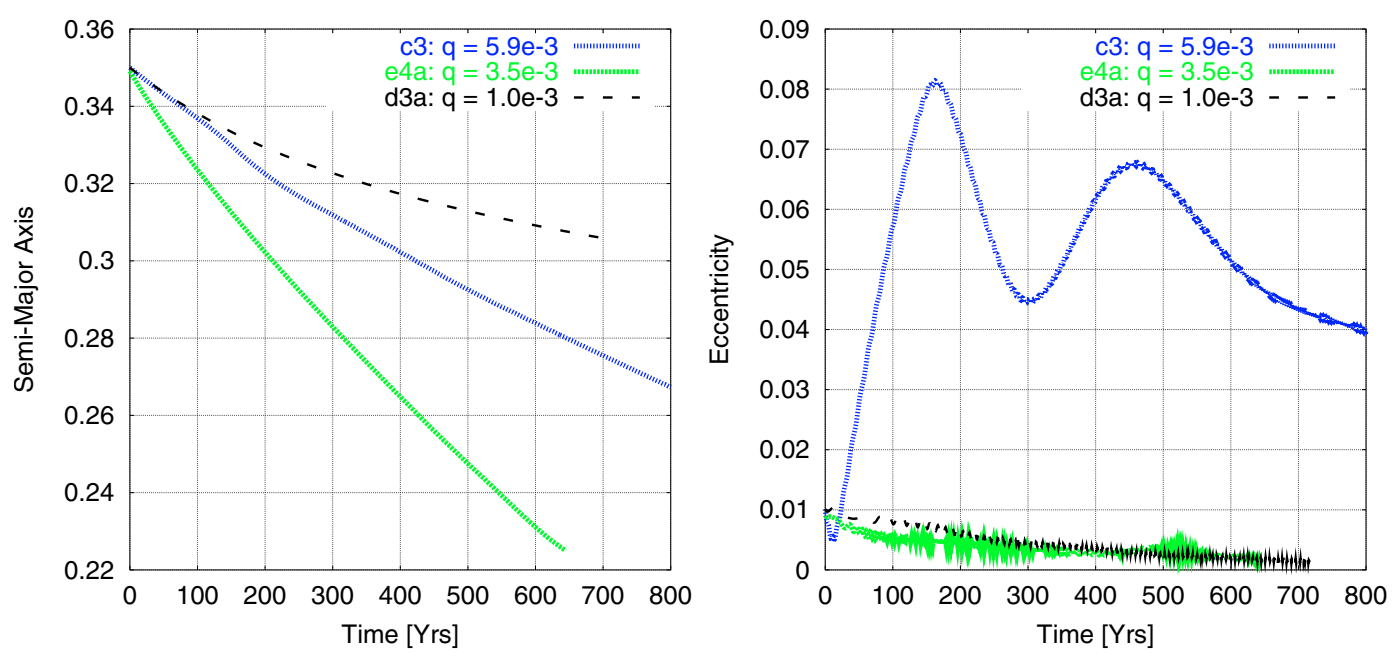

Fig. 5. The time evolution of the semi-major axis and eccentricity for three models with different masses of the outer planet, and zero mass inner planet.

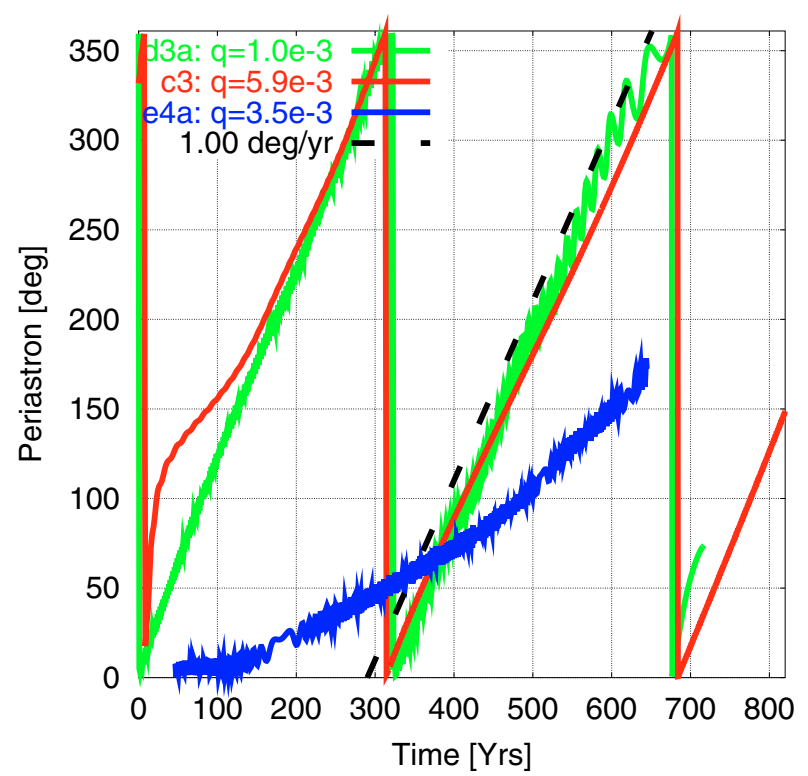

Fig. 6. The time evolution of the periastron for three models with different masses of the outer planet. The thick dashed line is a fit corresponding to a 1 deg $\mathrm{yr}^{-1}$ shift.

the massive planet. However, due to the eccentric disk (Fig. 4), material gets close to the planet every orbit, which increases the migration rate slightly. Thus, the non-monotonic variation of migration speed with planet mass is related to the different equilibrium disk states, circular and eccentric. The right panel of Fig. 5 shows a temporary increase of the eccentricity followed by a decline for the larger mass planet case, while the smaller masses both show declining eccentricities at nearly the same rates indicating the standard eccentricity damping in planet-disk interaction. This different behavior of the eccentricity evolution is definitely caused by the eccentric disk state for the higher planet mass case. It remains to be studied if this effect may have some relation to the observed large eccentricities of the extrasolar planets.
In Fig. 6 the evolution of the periastron is displayed for the three cases. For comparison the superimposed black dashedline refers to a periastron advance of exactly $1^{\circ} \mathrm{yr}^{-1}$. This positive $\dot{\varpi}_{\text {disk }} \approx 1^{\circ} \mathrm{yr}^{-1}$ (for the small and high mass planet) is solely due to the interaction of the disk with outer planet alone. The amount of planetary precession induced by the presence of the disk is determined primarily by the material being very close to the planet. Here, the behaviour of the low and high mass models are comparable again, while for the intermediate mass, there is less material very close to the planet and the precession rate is smallest.

\subsubsection{Switched on inner planet}

In the second step of the relaxation process the inner planet is included, using $q_{1}=1.75 \times 10^{-3}$. Additionally, we use the full thermodynamics for some models. The effect that these additions have on the density and temperature distribution is displayed in Figs. 7 and 8. The surface density is slightly increased upon including the inner planet because the additional torques tend to push the matter a bit further away from the central star. The presence of the outer planet is now seen clearly in the surface density distribution because for these models the planet is not allowed to accrete material from its Roche-lobe. The subsequent accumulation of gas in the Roche-lobe appears as a spike near $r=0.35$ in Fig. 7. The inclusion of radiative diffusion (Eq. (5)) in the plane of the disk reduces the temperature in the region of the planet and leads to a larger mass accumulation in the Roche lobe of the planet than in the model which includes only heating and cooling (Eq. (1)). The density distribution in the disk and gap region are not influenced too much by including radiative diffusion.

In Fig. 8 the change in temperature due to the inclusion of radiative effects is displayed. Due to the relatively large density of disk and the high viscosity, the temperature in the radiative cases rise considerably above the isothermal case, and is given in the disk region by the equilibrium of heating and cooling $D=Q$. The disk thickness increases from $H / r=0.05$ 


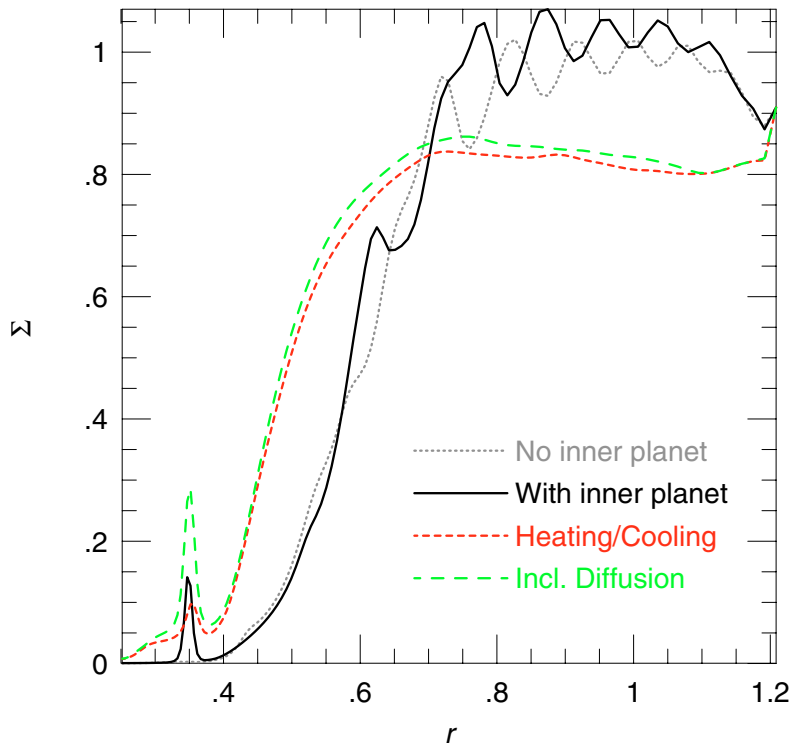

Fig. 7. The relaxed azimuthally averaged density profile for two isothermal models with and without considering the inner planet (solid, dotted lines), and two radiative models including only heating and cooling (short-dashed), and additionally radiative diffusion (longdashed). The mass of the inner planet is $q_{1}=1.75 \times 10^{-3}$ and that of the outer $q_{2}=5.9 \times 10^{-3}$. The gray short-dashed line is identical to the corresponding line in Fig. 3 (labeled 5.9).

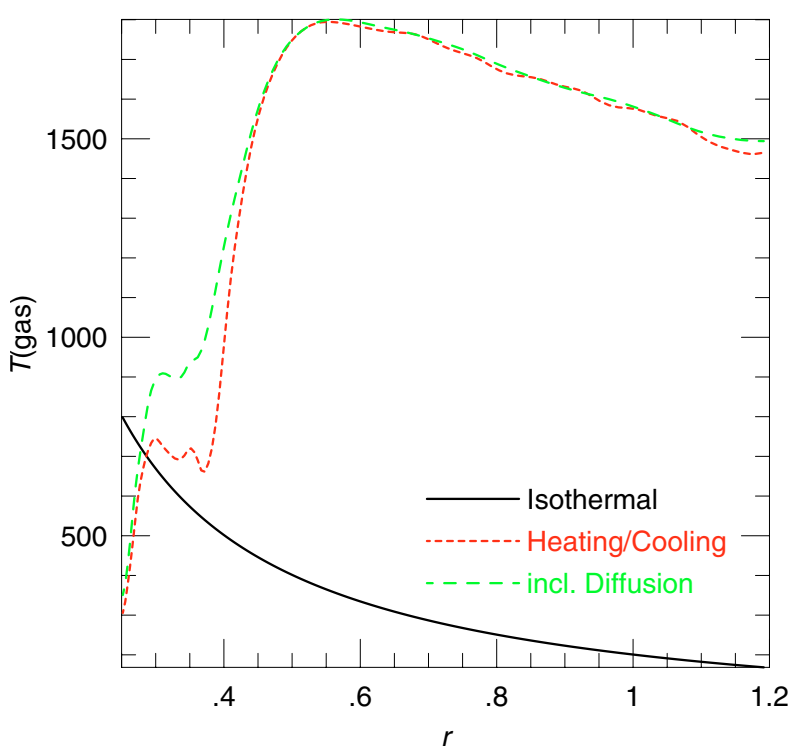

Fig. 8. The relaxed azimuthally averaged temperature profile for the isothermal (solid line) and two radiative models including only heating and cooling (short-dashed), and additionally radiative diffusion (long-dashed). The mass of the inner planet is $q_{1}=1.75 \times 10^{-3}$ and that of the outer $q_{2}=5.9 \times 10^{-3}$.

to about $H / r=0.15$, for the full radiative models. Including radiative diffusion in the plane of the disk (long-dashed line) leads to higher temperature in the gap region. The full thermodynamic models are no longer eccentric even for the large planetary mass. The included radiation and larger $H / r$ leads to a narrower gap and additional damping of the modes. We do not investigate at this point the detailed dependence of the disk thermodynamics on variations of the physical viscosity.
Table 2. Parameters of the models used to study the evolution of a planetary system under the action of an outer disk. Given are a model name, the used thermodynamics (TD), isothermal (iso) or radiative (rad), the type of outer boundary condition (obc) (open or closed), the mass-ratio of the inner planet $\left(q_{1}\right)$, the mass ratio or evolution of the outer planet $\left(q_{2}\right)$, the figure in which the model is displayed. The displayed radiative models include heating and cooling only as the inclusion of the diffusion does not change results significantly.

\begin{tabular}{ccclll}
\hline \hline Name & TD & obc & $\begin{array}{l}q_{1} \\
{\left[10^{-3}\right]}\end{array}$ & $\begin{array}{l}q_{2} \\
{\left[10^{-3}\right]}\end{array}$ & Fig. \\
\hline h4 & iso & closed & 1.75 & 3.50 & 9 \\
h2 & iso & closed & 1.75 & 5.9 & 10 \\
h9 & iso & closed & 2.10 & 7.10 & 11 \\
m1 & rad & closed & 1.75 & 5.9 & 12 \\
k3b & iso & open & 1.75 & 5.9 & 13 \\
h8a & iso & open & 2.10 & $5.9 \rightarrow 6.9$ & 14 \\
m2 & rad & open & 1.75 & 5.9 & 15 \\
\hline
\end{tabular}

These fully relaxed models including the inner planet serve as the starting point for the dynamical evolution of the planets. The periastron advance for the outer planet due to the inner planet having $q_{1}=0.00175$ and $a_{1}=0.20$ alone, with $n o$ disk forces is found to be $\dot{\varpi}_{\text {planet }} \approx 0.68^{\circ} \mathrm{yr}^{-1}$. Thus, the disk and the planet generate a shift of similar magnitude.

\subsection{Evolving planets}

Having constructed several equilibrium models holding the orbital elements of the planets fixed, we now release the planets and follow the evolution of their orbital elements. For an overview Table 2 lists the models and their most important parameters.

In the first instance we keep the damped and reflecting boundary conditions at the outer boundary, i.e. we model the situation where the disk remains in full contact with the planet during the evolution. In Figs. 9 to 11 we display the evolution of the semi-major axes, the eccentricities, and the two resonant angles $\theta_{1}$ and $\Delta \varpi$ for three models, a test case (model h4) with a lighter outer planet $\left(q_{1}=0.00175, q_{2}=0.0035\right)$, a case (h2) resembling the edge-on $\left(q_{1}=0.00175, q_{2}=0.0059\right)$ system, and finally the $i=55^{\circ}\left(q_{1}=0.0021, q_{2}=0.0071\right)$ case (h9), respectively. In all cases the outer planet captures the inner one into a 2:1 resonance. Although the orbits are initially quite far from 2:1 mean-motion commensurability, the resonant angle $\theta_{1}$ is already in libration, with amplitude as large as $\sim 90^{\circ}$. However, this libration of $\theta_{1}$ has little dynamical consequence and the increase in $e_{1}$ is slow, until the mean motions approach the $2: 1$ commensurability. There is a delay in the capture of $\Delta \varpi$ (and $\theta_{2}$ ) into resonance, and the libration amplitude of $\theta_{1}$ is smaller than that of $\Delta \varpi$. Note that in the first test-case for smaller planet mass, when the initial disk state was not eccentric, the capture of $\Delta \varpi$ occurs faster and the libration amplitudes of both $\theta_{1}$ and $\Delta \varpi$ are much smaller.

The fact that $\theta_{1}$ (and even $\theta_{2}$ and $\Delta \varpi$ ) can be librating when the orbital mean-motions are far from the 2:1 commensurability is due to the $1 / e_{j}$ dependence of the resonance-induced retrograde precession of $\varpi_{j}$ at small $e_{j}$. The relative timing of the capture of $\theta_{1}$ and $\theta_{2}$ into resonance is affected by the masses 

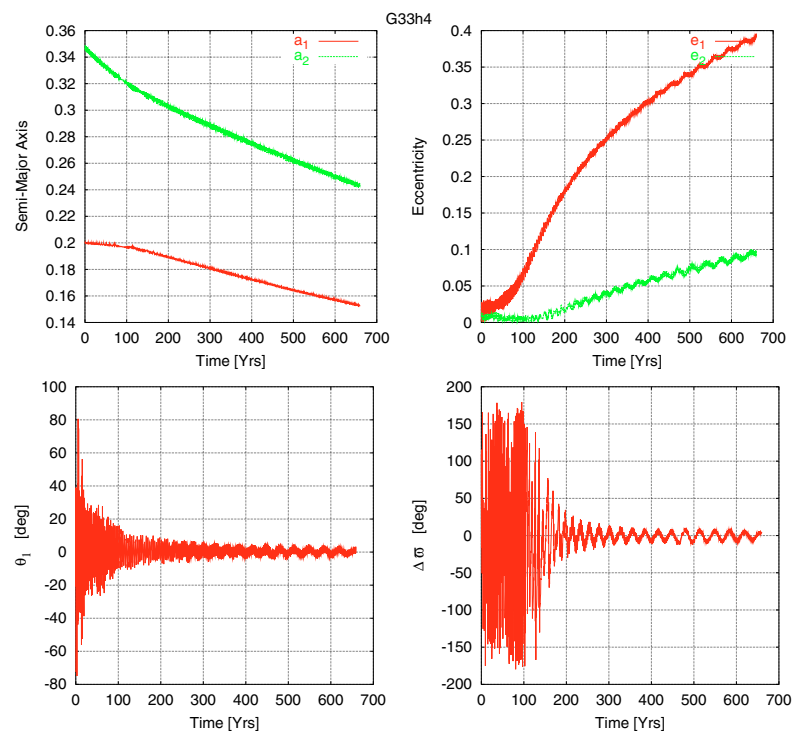

Fig. 9. The time evolution of the orbital elements $\left(a, e, \theta_{1}, \Delta \varpi\right)$ for an isothermal model (h4) with $q_{1}=1.75 \times 10^{-3}$ and $q_{2}=3.5 \times 10^{-3}$ (test case with lower outer planet mass).
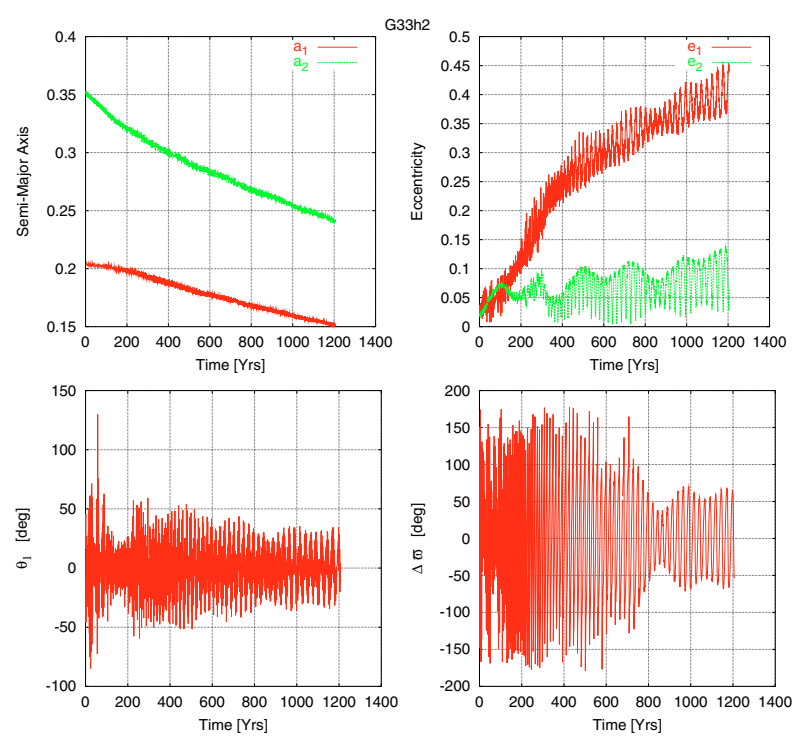

Fig. 10. The time evolution of the orbital elements $\left(a, e, \theta_{1}, \Delta \varpi\right)$ for an isothermal model (h2) with $q_{1}=1.75 \times 10^{-3}$ and $q_{2}=5.9 \times 10^{-3}$ (as GJ 876 edge on).

and initial eccentricities of the planets. We shall discuss these points further in Sect. 5.

Once both $\theta_{1}$ and $\Delta \varpi$ are librating about $0^{\circ}$, the eccentricities rise rapidly. In cases h2 and h9, applicable to GJ 876, the eccentricity of the inner planet rises above 0.4 , which clearly exceeds the upper limit of $\sim 0.31$ for GJ 876 (see Fig. 1).

The evolution with the inclusion of heating and cooling (m1) is displayed in Fig. 12. Again the system is caught in a 2:1 resonance, and the eccentricities rise to high values. Here, due to the smoother initial state, the alignment of the resonant angles is much stronger and the libration amplitudes for both angles are reduced strongly. The evolution beyond $t=380 \mathrm{yr}$ is unreliable because both planets are outside the computational domain by then. The models with radiative diffusion do not
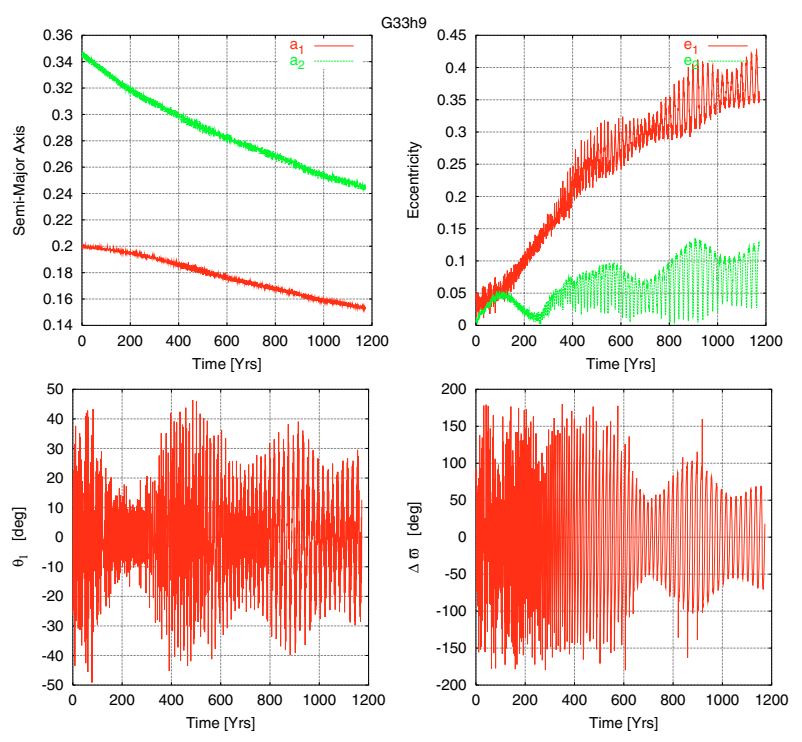

Fig. 11. The time evolution of the orbital elements $\left(a, e, \theta_{1}, \Delta \varpi\right)$ for an isothermal model (h9) with $q_{1}=2.10 \times 10^{-3}$ and $q_{2}=7.1 \times 10^{-3}$ (as GJ 876 at $55 \mathrm{deg}$ inclination).
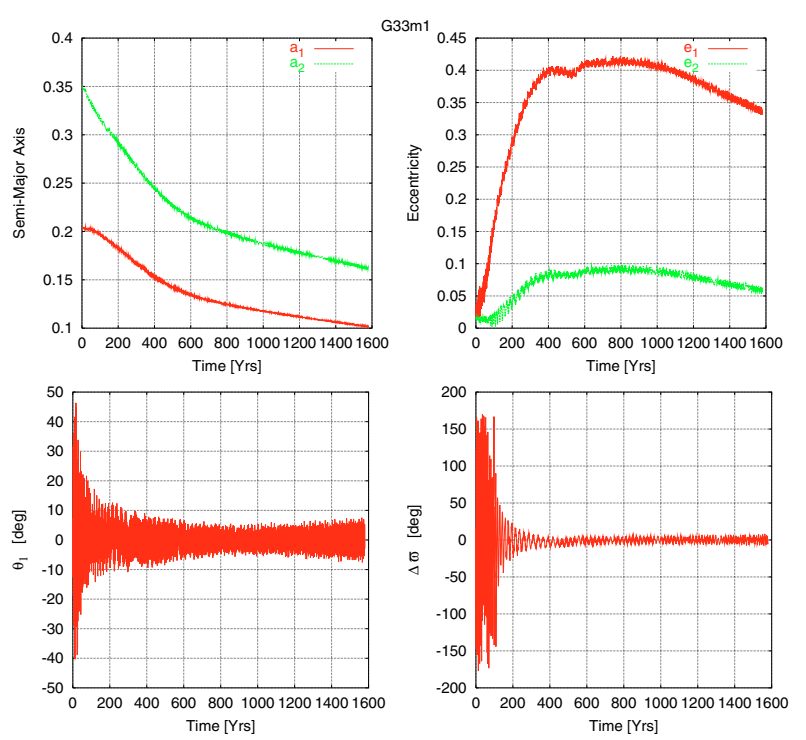

Fig. 12. The time evolution of the orbital elements $\left(a, e, \theta_{1}, \Delta \varpi\right)$ for a radiative model including heating and cooling $(\mathrm{m} 1)$ with $q_{1}=1.75 \times$ $10^{-3}$ and $q_{2}=5.9 \times 10^{-3}$ (as GJ 876 edge on). Note that at around $t=380$ the outer planet leaves the computational grid and the results are not reliable anymore.

yield any different results from models with only heating and cooling. The equilibrium configurations as shown in Figs. 7 and 8 above are very similar already.

In summary, there exist two different types of evolutions of the orbital elements depending on the state of disk. For a nearly circular disk (models $\mathrm{h} 4, \mathrm{~m} 1$ ) the delay in the capture of $\Delta \varpi$ (or equivalently $\theta_{2}$ ) allows $e_{2}$ to be initially damped. After capture in this second resonance $e_{2}$ rises rapidly and increases far beyond the upper limit inferred from the observations. The libration of the resonant angles after capture is very small. This result confirms earlier findings of hydrodynamical evolutions of resonant planets (Kley et al. 2004). For an eccentric disk 

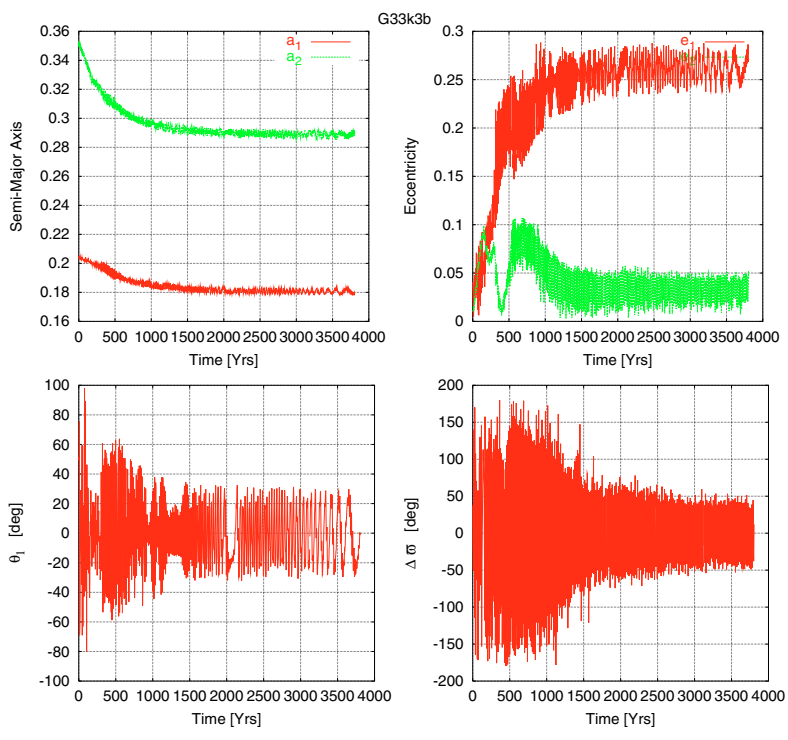

Fig. 13. The time evolution of the orbital elements $\left(a, e, \theta_{1}, \Delta \varpi\right)$ for an isothermal model $(\mathrm{k} 3 \mathrm{~b})$ with $q_{1}=1.75 \times 10^{-3}$ and $q_{2}=5.9 \times 10^{-3}$, including disk dispersal.

state, i.e. for larger outer planet masses, capture of $\theta_{2}$ is more delayed and the libration of the two resonant angles remains quite large. Nevertheless, the eccentricities quickly exceed the upper limits for GJ 876.

\subsubsection{Modeling disk dispersal}

The next set of models starts with the same conditions as the previous ones but allows for material to leave the outer boundary; this could occur if the outer disk is photoevaporated, for example. Depending on the maximum outflow velocity allowed, the mass in the disk can be reduced slowly or very efficiently. For our purposes we set the velocity in the outer radial ghostcell (the gridcell just beyond $R_{\max }$ ) to $1 / 10$ of the value of the adjacent inner gridcell. This results in a mass loss rate which is somewhat higher than physically plausible, but allows us to halt the migration in a computationally feasible time. For the isothermal models (k3b) and (h8) we find a masshalf-emptying time of about $300 \mathrm{yr}$ and for the radiative model $100 \mathrm{yr}$.

In Figs. 13 and 14 two isothermal cases are presented; in the first $(\mathrm{k} 3 \mathrm{~b})$, the mass of the outer planet remains constant, while in the second model (h8a) the planet is allowed to accrete from its surroundings and to grow in mass. In the second case the mass of the inner planet was chosen higher $q_{1}=0.0021$ to compensate for the higher final mass of the outer planet. Again, capture into the $2: 1$ resonance occurs, but the eccentricities do not rise to such high values. The loss of mass from the disk turns the migration off before the eccentricities get large. The alignment of the resonant angles occurs on longer timescales than before. Similar behavior can be seen in the evolution of the radiative model (m2) displayed in Fig. 15. Again the alignment of the resonant angles occurs faster and libration of the resonant angles is smaller than in the isothermal models due to the noneccentric disk state.
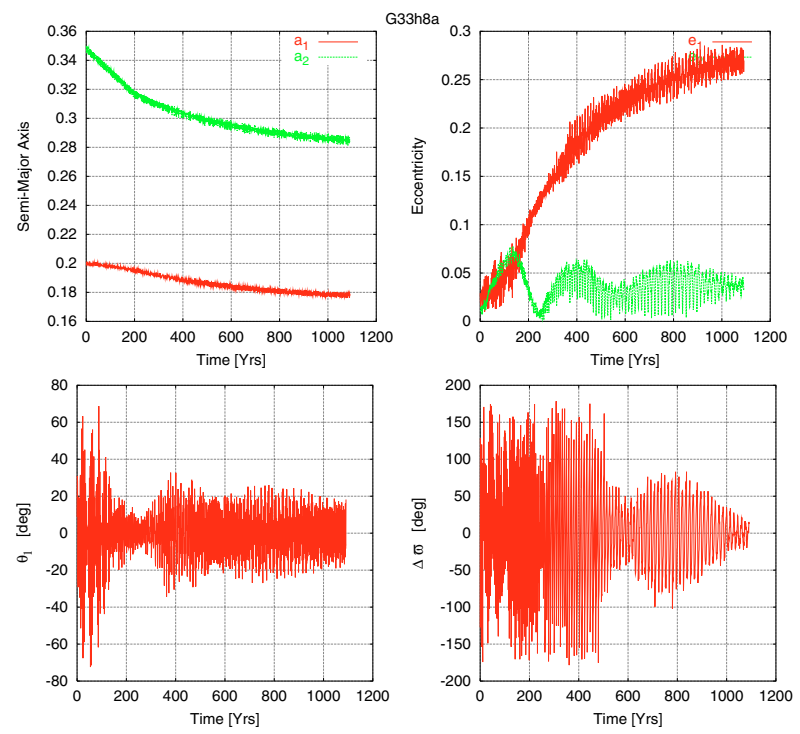

Fig. 14. The time evolution of the orbital elements $\left(a, e, \theta_{1}, \Delta \varpi\right)$ for an isothermal model (h8a) with $q_{1}=2.10 \times 10^{-3}$ and a growing outer planet $q_{2}=5.9 \rightarrow 6.9 \times 10^{-3}$. The model includes disk dispersal.
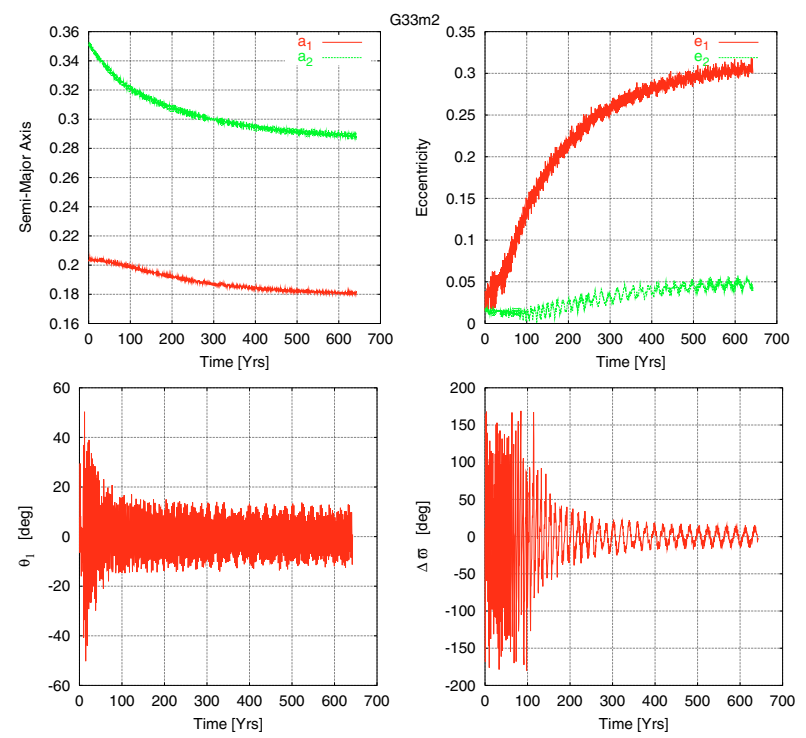

Fig. 15. The time evolution of the orbital elements $\left(a, e, \theta_{1}, \Delta \varpi\right)$ for a radiative model $(\mathrm{m} 2)$ with $q_{1}=1.75 \times 10^{-3}$ and $q_{2}=5.9 \times 10^{-3}$, including disk dispersal.

\section{Interpretations}

In this section we use three-body integrations and analytic theory to interpret the hydrodynamic results presented in Sect. 4. The three-body integrations were performed using the symplectic integrator SyMBA (Duncan et al. 1998), modified to include forced migration and apsidal precession and to have input and output in Jacobi coordinates (Lee \& Peale 2002; Lee 2004). Although three-body integrations do not model the interactions between the disk and the planets self-consistently, they allow different effects to be included separately to assess their importance. In particular, we show that the disk-induced apsidal precession, which has not been included in previous three-body integrations, does not cause an additional delay in 

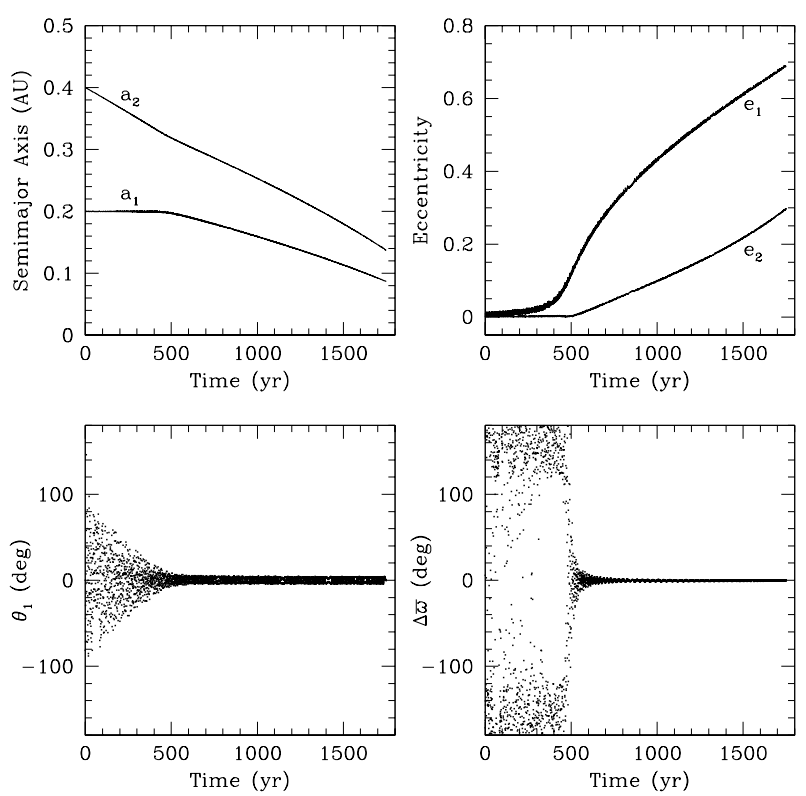

Fig. 16. Time evolution of the orbital elements $\left(a, e, \theta_{1}, \Delta \varpi\right)$ for the baseline three-body model with $q_{1}=1.75 \times 10^{-3}$ and $q_{2}=5.9 \times$ $10^{-3}$. The planets are initially on coplanar circular orbits. The outer planet is forced to migrate inward at the rate $\dot{a}_{2} / a_{2}=-4.8 \times$ $10^{-4}\left(0.35 \mathrm{AU} / a_{2}\right)^{3 / 2} \mathrm{yr}^{-1}$, and there is no eccentricity damping or additional apsidal precession.

the capture of $\theta_{2}$ and $\Delta \varpi$, and that the additional delay in the capture of $\Delta \varpi$ in, e.g., the isothermal models h2 (Fig. 10) and h9 (Fig. 11) is due to the relatively large initial eccentricities induced by the eccentric disk.

Figure 16 shows the evolution of the semimajor axes, eccentricities, and resonant angles $\theta_{1}$ and $\Delta \varpi$ for the baseline three-body model with $q_{1}=1.75 \times 10^{-3}$ and $q_{2}=5.9 \times 10^{-3}$. The outer planet is forced to migrate inward at the rate $\dot{a}_{2} / a_{2}=$ $-4.8 \times 10^{-4}\left(0.35 \mathrm{AU} / a_{2}\right)^{3 / 2} \mathrm{yr}^{-1}$ (which matches the migration rate at $a_{2}=0.35 \mathrm{AU}$ for the h2 model in Fig. 10), and there is no eccentricity damping or additional apsidal precession. The planets are initially on coplanar circular orbits, with $a_{1}=0.2 \mathrm{AU}$ and $a_{2}=0.4 \mathrm{AU}$. The circular orbits and larger $a_{2}$ compared to the hydrodynamical simulations (where $\left.a_{2}=0.35 \mathrm{AU}\right)$ reduce the initial eccentricity variations. As in the hydrodynamical simulations, the resonant angle $\theta_{1}$ is already librating about $0^{\circ}$ at the start of the evolution, even though the planets are started even further from the $2: 1$ meanmotion commensurability than in the hydrodynamical simulations. With the small initial eccentricity variations and the use of Jacobi coordinates, it can be seen in Fig. 16 that $\Delta \varpi$ (and $\theta_{2}$ ) are first captured into libration about $180^{\circ}$ at $t \approx 270 \mathrm{yr}$ and that $\Delta \varpi$ spends more time around $180^{\circ}$ even before the capture. The system passes smoothly over to the configuration with both $\theta_{1}$ and $\Delta \varpi$ librating about $0^{\circ}$ when $e_{1} \gtrsim 0.1$ (see also Lee 2004). The small libration amplitudes of the configuration with both $\theta_{1}$ and $\Delta \varpi$ librating about $0^{\circ}$ are similar to those found in the radiative model $\mathrm{m} 1$ (Fig. 12).
For two planets orbiting a star in coplanar orbits, the equations of motion for the periapse longitude and eccentricity are

$$
\begin{aligned}
\frac{\mathrm{d} \varpi_{j}}{\mathrm{~d} t}= & -\frac{\sqrt{1-e_{j}^{2}}}{M_{j} e_{j} \sqrt{G M_{*} a_{j}}} \frac{\partial \Phi}{\partial e_{j}}+\dot{\varpi}_{\mathrm{sec}, j}+\dot{\varpi}_{\mathrm{disk}, j} \\
\frac{\mathrm{d} e_{j}}{\mathrm{~d} t}= & \frac{\sqrt{1-e_{j}^{2}}}{M_{j} e_{j} \sqrt{G M_{*} a_{j}}} \frac{\partial \Phi}{\partial \varpi_{j}} \\
& -\frac{\left(1-e_{j}^{2}\right)-\sqrt{1-e_{j}^{2}}}{M_{j} e_{j} \sqrt{G M_{*} a_{j}}} \frac{\partial \Phi}{\partial \lambda_{j}}+\dot{e}_{\mathrm{disk}, j},
\end{aligned}
$$

where the disturbing potential

$\Phi=-\frac{G M_{1} M_{2}}{a_{2}} \varphi\left(\beta, e_{1}, e_{2}, \theta_{1}, \theta_{2}\right)$,

the ratio $a_{1} / a_{2}=\beta$, and $\varphi$ is a function of the indicated variables if we consider only the $2: 1$ resonant terms and neglect terms of order $\left[\left(M_{1}+M_{2}\right) / M_{*}\right]^{2}$ and higher (e.g. Yoder \& Peale 1981; Lee \& Peale 2002; Beaugé \& Michtchenko 2003). The term $\dot{\varpi}_{\text {disk, } j}$ represents the positive apsidal precession induced by the disk, $\dot{\varpi}_{\mathrm{sec}, j}$ the direct secular effect from the other planet, and $\dot{e}_{\text {disk, } j}$ the eccentricity damping induced by the disk. The disk-induced variations are not included in Fig. 16, and we neglect them in our discussion for the moment.

When the eccentricities are very small, the relevant terms in $\varphi$ are $C_{1}(\beta) e_{1} \cos \theta_{1}$ and $C_{2}(\beta) e_{2} \cos \theta_{2}$, and we have

$$
\begin{aligned}
& \mathrm{d} \varpi_{1} / \mathrm{d} t=\beta q_{2} n_{1} C_{1} e_{1}^{-1} \cos \theta_{1}+\dot{\varpi}_{\mathrm{sec}, 1}, \\
& \mathrm{~d} \varpi_{2} / \mathrm{d} t=q_{1} n_{2} C_{2} e_{2}^{-1} \cos \theta_{2}+\dot{\varpi}_{\mathrm{sec}, 2}
\end{aligned}
$$

and

$\mathrm{d} e_{1} / \mathrm{d} t=-\beta q_{2} n_{1} C_{1} \sin \theta_{1}$,

$\mathrm{d} e_{2} / \mathrm{d} t=-q_{1} n_{2} C_{2} \sin \theta_{2}$,

where $n_{j}$ are the mean motions and $C_{1}(\beta) \approx-1.19$ and $C_{2}(\beta) \approx$ +0.43 for $\beta \approx 2^{-2 / 3}$ (Yoder \& Peale 1981). Stable simultaneous librations of both $\theta_{1}$ and $\theta_{2}$ (or equivalently $\Delta \varpi$ ) require that the longitudes of the periapses regress at the same rate on average and that the eccentricities do not change in the absence of continued migration. Since $C_{1}<0$ and $C_{2}>0$, the only way these requirements are satisfied is for $\theta_{1}$ to librate about $0^{\circ}$ and $\theta_{2}$ and $\Delta \varpi$ to librate about $180^{\circ}$. This anti-aligned configuration is what we observe with the Io-Europa pair of Jupiter's satellites and is shown in Fig. 16 just after the capture of $\Delta \varpi$.

The relative timing of the capture of $\theta_{1}$ and $\theta_{2}$ into resonance needs some clarification. Due to the $1 / e_{1}$ dependence of the resonance-induced precession of $\varpi_{1}$ at small $e_{1}$ (Eq. (11)), a small initial value of $e_{1}$ leads to an extremely mobile longitude of periapse $\varpi_{1}$, such that the large mass of the outer planet can induce sufficient retrograde motion of $\varpi_{1}$ to cause $\theta_{1}$ to already be librating at the start of the evolution when the orbital mean motions are far from the 2:1 commensurability. This initial libration is evident in the three-body model shown in Fig. 16 and in the hydrodynamical simulations shown in Sect. 4.2. It should be noted, however, that this libration of $\theta_{1}$ has little dynamical consequence and the increase in $e_{1}$ (see next paragraph) is slow, 
until the mean motions approach the 2:1 commensurability. In spite of the relatively small initial value of $e_{2}$ in Fig. 16 and the same $1 / e$ dependence of the resonance-induced precession (Eq. (12)), $\theta_{2}$ and hence $\Delta \varpi$ are not librating initially, although they do spend more time near $180^{\circ}$. This is due to the inner planet's lower mass being insufficient to perturb the more massive outer planet's $\varpi_{2}$ enough to cause $\theta_{2}$ to librate that far from the $2: 1$ commensurability of the mean motions.

The reason the eccentricities increase with continued migration can be understood as follows. Continued inward migration of the outer planet while the system is within the resonances means $a_{2}$ is consistently slightly smaller than it would have been without the migration. The increased value of $n_{2}$ therefore means $\lambda_{2}$ is slightly larger at any instant than it would be without the migration. The argument of the sine in Eq. (13), $\theta_{1}=2 \lambda_{2}-\lambda_{1}-\varpi_{1}$, is then slightly greater than $0^{\circ}$ on average. Since $C_{1}<0, \mathrm{~d} e_{1} / \mathrm{d} t>0$ and the eccentricity must grow. A similar argument applies to $\mathrm{d} e_{2} / \mathrm{d} t$ if $\theta_{2}$ is also librating about $180^{\circ}$ (see Eq. (14)). It can be shown from the equations for the evolution of the orbital energy and angular momentum that at least one, but not necessarily both, of the eccentricities must be increasing at any given time for continued migration within the resonance, if $e_{2}^{2}<\left(3+e_{1}^{2}\right) / 4$ for the $2: 1$ resonance and there is no eccentricity damping (see Eq. (A10) of Murray et al. 2002).

For $M_{1} / M_{2} \approx 0.31$, the resonant interaction is no longer dominated by the lowest order resonant terms when $e_{1} \gtrsim 0.04$, and $e_{2}$ changes from increasing to decreasing with continued migration until $e_{2} \approx 0$ when $e_{1} \approx 0.1$. This change in the evolution of $e_{2}$ is not obvious in Fig. 16 because $e_{2} \lesssim 0.002$ during this phase; see Fig. 5 of Lee (2004). Then the libration center of $\theta_{2}$ and $\Delta \varpi$ changes from $180^{\circ}$ to $0^{\circ}$ (with a slight phase shift due to the continued migration), and both eccentricities continue to grow because of the slight phase shift in the arguments of the terms involving linear combinations of $\theta_{1}$ and $\theta_{2}$. However, because there are now many terms in the disturbing potential that contribute to the resonant interaction, a simple demonstration of the co-precession of the periapses with both $\theta_{1}$ and $\theta_{2}$ librating about $0^{\circ}$ and of the increase in both eccentricities with continued migration is elusive. Continued migration causes the eccentricities to quickly exceed those observed without a large eccentricity damping as discussed above. Although the hydrodynamical simulations self-consistently produce $\dot{e}_{\text {disk }}<0$, the magnitude of this damping is far less than that necessary to maintain the small observed eccentricities in the GJ 876 system during continued migration.

\subsection{Disk-induced apsidal precession}

It is shown in Fig. 6 that the disk induces a prograde apsidal precession of the outer planet of about $1^{\circ} \mathrm{yr}^{-1}$ for $q_{2}=$ $5.9 \times 10^{-3}$. This prograde apsidal precession is primarily due to the axisymmetric component of the disk potential. For a planet with orbital semimajor axis $a$ and mean motion $n$, an outer disk with surface mass density $\Sigma(r)=\Sigma_{0}\left(r_{0} / r\right)^{k}$ at $r_{1}<r<r_{2}$

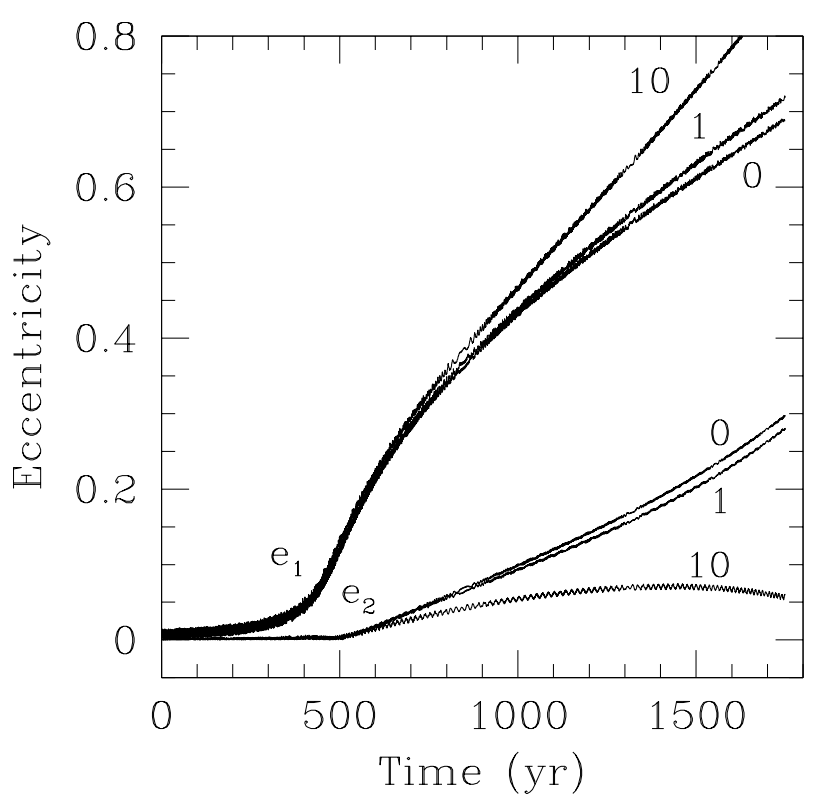

Fig. 17. Time evolution of the orbital eccentricities for the three-body models with additional apsidal precession $\dot{\varpi}_{\text {disk,2 }}=$ $p\left(0.35 \mathrm{AU} / a_{2}\right)^{3 / 2}{ }^{\circ} \mathrm{yr}^{-1}$ and $p=1$ and 10 , compared to the case without additional apsidal precession $(p=0)$ from Fig. 16.

(where $r_{1}>a$ ) induces a prograde apsidal precession (Ward 1981)

$\dot{\varpi}_{\text {disk }}=2 \pi n\left(\frac{\Sigma_{0} a^{2}}{M_{*}}\right)\left(\frac{r_{0}}{a}\right)^{k}\left[W_{k}\left(r_{1} / a\right)-W_{k}\left(r_{2} / a\right)\right]$.

where

$W_{k}(r / a)=\sum_{\ell=1}^{\infty} \frac{\ell(2 \ell+1)}{(2 \ell+k-1)}\left[\frac{(2 \ell) !}{2^{2 \ell}(\ell !)^{2}}\right]^{2}\left(\frac{a}{r}\right)^{2 \ell+k-1}$.

If we approximate the azimuthally averaged density profile for $q_{2}=5.9 \times 10^{-3}$ in Fig. 3 as a flat profile $(k=0)$ with $\Sigma_{0}=7500 \mathrm{~g} \mathrm{~cm}^{-2}$ between $r_{1}=0.6 \mathrm{AU}$ and $r_{2}=1.2 \mathrm{AU}$, we find $\dot{\varpi}_{\text {disk,2 }} \approx 0.7 \mathrm{yr}^{-1}$, which is in reasonable agreement with the measured value. The disk-induced precession rate can be increased by increasing the disk mass or decreasing the gap width $\left(r_{1}-a\right) / a$, but the gap width is determined mainly by the mass ratio $q$ and is not very sensitive to either the viscosity parameter $\alpha$ or the disk thickness $H / r$ (e.g. Kley 1999; Bryden et al. 1999; Varnière et al. 2004). Increasing $\alpha$ and/or $H / r$ primarily makes the gap shallower and not so deep.

We have performed three-body integrations similar to that in Fig. 16, but with additional apsidal precession $\dot{\varpi}_{\text {disk, } 2}=$ $p\left(0.35 \mathrm{AU} / a_{2}\right)^{3 / 2}{ }^{\circ} \mathrm{yr}^{-1}$ and $p=1$ and 10 . The much smaller disk-induced precession of the inner planet's orbit was neglected. Except for a larger libration amplitude for $\Delta \varpi$ in the $p=10$ case, the evolution of $\theta_{1}$ and $\Delta \varpi$ is similar to that shown in Fig. 16 for $p=0$, and there is no additional delay in the capture of $\Delta \varpi$. Figure 17 shows the evolution of the eccentricities for $p=0,1$, and 10. At a given time, the additional positive apsidal precession results in an increase in $e_{1}$ and a decrease in $e_{2}$, but the effect is small for $e_{1}<0.31$ (the upper limit for GJ 876 ; Fig. 1) even for $p=10$. The additional apsidal precession and forced migration rates used in the three-body integrations 
are proportional to $a_{2}^{-3 / 2}$ and hence the mean motion $n_{2}$. A decrease in the positive, disk-induced precession rate due to, e.g., disk dispersal would cause the eccentricities to adjust to values appropriate for the precession rate at a given time and approach the $p=0$ case without disk-induced precession.

The analytic theory developed by Yoder \& Peale (1981) for the 2:1 resonances of Io and Europa and the Laplace resonance takes into account the apsidal precession induced by the oblateness of Jupiter (which is largest for the innermost satellite Io), and it can be adapted to understand the effects of the disk-induced apsidal precession (which is larger for the outer planet). As the orbits converge toward the $2: 1$ mean-motion commensurability (i.e., as $2 n_{2}-n_{1}<0$ increases), the resonance $\theta_{1}=2 \lambda_{2}-\lambda_{1}-\varpi_{1}$ would be encountered before the resonance $\theta_{2}=2 \lambda_{2}-\lambda_{1}-\varpi_{2}$ if the apsidal precession is dominated by $\dot{\varpi}_{\text {disk, } j}$ (since $\dot{\theta}_{j} \approx 2 n_{2}-n_{1}-\dot{\varpi}_{j}$ and $\dot{\varpi}_{\text {disk }, 2} \gg \dot{\varpi}_{\text {disk, } 1}>0$ ). However, because the resonance-induced retrograde apsidal precession is proportional to $1 / e_{j}$ and much larger in magnitude than the disk-induced prograde precession for small $e_{j}, \theta_{1}$ and $\theta_{2}$ (and hence $\Delta \varpi$ ) can be captured into libration in a sequence that differs little in order or timing from the case where there is no disk-induced precession. When we include $\dot{\varpi}_{\text {disk, } j}$ in Eqs. (11) and (12) for small $e_{j}$, stable retrograde precessions of both orbits still require $\left\langle\theta_{1}\right\rangle=0^{\circ}$ and $\left\langle\theta_{2}\right\rangle=180^{\circ}$, and the requirement that the orbits precess at the same rate on average $\left(\left\langle\dot{\varpi}_{1}\right\rangle=\left\langle\dot{\varpi}_{2}\right\rangle\right)$ implies the following relationship between the forced eccentricities:

$$
\begin{aligned}
e_{2} / e_{1}= & q_{1} n_{2} C_{2} /\left[-\beta q_{2} n_{1} C_{1}+e_{1}\left(\dot{\varpi}_{\mathrm{sec}, 2}-\dot{\varpi}_{\mathrm{sec}, 1}\right)\right. \\
& \left.+e_{1}\left(\dot{\varpi}_{\mathrm{disk}, 2}-\dot{\varpi}_{\mathrm{disk}, 1}\right)\right] .
\end{aligned}
$$

Since $\dot{\varpi}_{\text {disk }, 2}-\dot{\varpi}_{\text {disk, } 1}>0$, the disk-induced apsidal precession reduces $e_{2} / e_{1}$. The decrease in $e_{2}$ and increase in $e_{1}$ seen in Fig. 17 are consistent with this trend, but it should be noted that the $2: 1$ resonance configurations with $M_{1} / M_{2} \approx 0.3$ and $e_{1} \gtrsim 0.04$ are no longer dominated by the lowest order resonant terms and those with $e_{1} \gtrsim 0.1$ also have $\theta_{2}$ and $\Delta \varpi$ librating about $0^{\circ}$ instead of $180^{\circ}$ (see above). Although $e_{2} / e_{1}$ is fixed by the resonance conditions, a simple expression like Eq. (17) does not exist for the larger eccentricities.

\subsection{Initial eccentricities}

The isothermal model h2 (Fig. 10) shows large initial eccentricity variations induced by the eccentric disk, with both $e_{1}$ and $e_{2}$ around 0.05 . The large eccentricities (in particular $e_{2}$ ) mean that the planets cannot be captured into the 2:1 resonance configuration with $\theta_{1}$ librating about $0^{\circ}$ and $\theta_{2}$ librating about $180^{\circ}$ (see Fig. 16). In Fig. 18 we show the results of a threebody integration similar to the baseline model in Fig. 16, but with the following initial conditions: $e_{1}=0.01, e_{2}=0.05$, and the orbits are antialigned, with the inner planet at periapse and the outer planet at apoapse. These initial conditions were chosen so that the eccentricity variations are similar to those in the model h2 when $a_{2} \approx 0.35 \mathrm{AU}$. The larger value of $e_{1}$ has prevented the initial libration of $\theta_{1}$ that is seen in Fig. 16. But $\theta_{1}$ is captured into libration about $0^{\circ}$ at about the time $(t \approx 300 \mathrm{yr})$ when $a_{2} \approx 0.35 \mathrm{AU}$, and its libration amplitude is similar to that
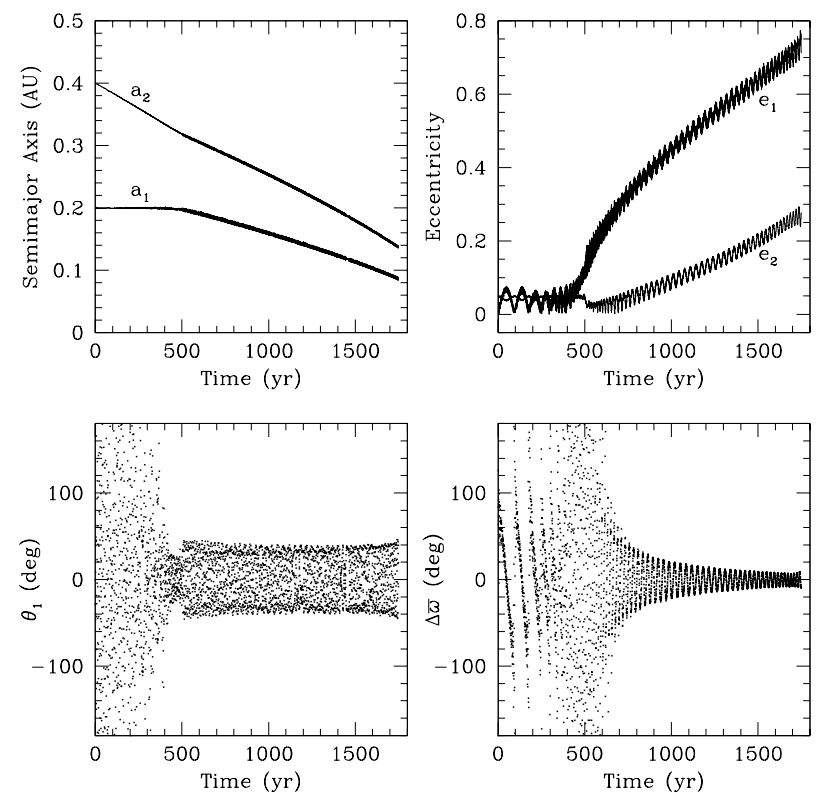

Fig. 18. Same as Fig. 16, but for the three-body model with the following initial conditions: $e_{1}=0.01, e_{2}=0.05$, and the orbits are antialigned, with the inner planet at periapse and the outer planet at apoapse.

in model h2. There is also a delay in the capture of $\Delta \varpi$ (and $\left.\theta_{2}\right)$ into libration to $t \approx 600 \mathrm{yr}$ and, as expected, $\Delta \varpi$ is captured directly into libration about $0^{\circ}$. The libration amplitude of $\Delta \varpi$ is smaller than that in model h2, possibly because of continuing interaction with an eccentric disk in the latter case. The relative timing of the captures can have no effect on the necessity of large eccentricity damping if there is extensive migration within the resonances, since the ratio of the eccentricities is determined uniquely by the resonance conditions once all the resonance variables are librating.

\section{Conclusion}

We have modeled the evolution of the GJ 876 system by performing two-dimensional hydrodynamical simulations of a disk with embedded planets. We confirm previous work showing that interactions between the outer planet and a gas disk can drive two initially non-resonant planets into resonance, and in particular we have shown that the induced migration of the GJ 876 planets results in the capture of the $2: 1$ resonance variables, $\theta_{1}, \theta_{2}$ and $\Delta \varpi$ into the observed libration about $0^{\circ}$. The precession of the outer planet's periapse longitude induced by the disk was shown not to increase the delay of capture of $\theta_{2}$ into resonance, but a high value of $e_{2}$ induced by an eccentric disk did increase the delay. Later capture of $\theta_{2}$ has no effect on the eccentricity damping problem as the ratio of the forced eccentricities is fixed once both $\theta_{1}$ and $\theta_{2}$ are librating.

The "isothermal" simulations with fixed disk temperature structure have shown that more massive planets with a planetto-star mass ratio of $q \geq 5.2 \times 10^{-3}$ are able to perturb the disk sufficiently to make it eccentric (even if the planetary orbit is circular). This effect may be caused by an eccentric instability driven by an interaction of the $m=2$ mode at the outer 
eccentric 3:1 Lindblad resonance with a slightly eccentric disk (see Papaloizou et al. 2001). For smaller masses in the isothermal models and for all masses in the radiative models, the planet-disk interaction produces the typical gap and spiral arms, but the disk remains otherwise circular.

The simulations also confirm that for non-eccentric, circular disks the eccentricity growth of the outer planet is suppressed until $\theta_{2}$ is locked into libration about $0^{\circ}$ (see models $\mathrm{h} 4$, $\mathrm{m} 1, \mathrm{~m} 2$ ). However, $\theta_{2}$ and $\Delta \varpi$ are always eventually captured into libration, and the subsequent increase in the eccentricities of both planets past the observational upper limits in GJ 876 $\left(e_{1} \approx 0.31, e_{2} \approx 0.05\right)$ occurs on a time scale shorter than the expected viscous time scale of the disk. The final eccentricities of both planets are then substantially larger than those seen in GJ 876, a result which was found already in previous hydrodynamic (Papaloizou 2003; Kley et al. 2004) and three-body simulations (e.g. Lee \& Peale 2002).

In addition to causing an increased delay in the capture of $\theta_{2}$ and $\Delta \varpi$, eccentric disks (models $\mathrm{h} 2, \mathrm{~h} 9$ ) lead to significantly larger libration amplitudes for $\Delta \varpi\left(\approx 50^{\circ}\right)$ than for the circular disk case. Interestingly, it appears that in the case of GJ 876 the libration of $\Delta \varpi$ is in fact larger $\left(\approx 34^{\circ}\right.$ for $\left.i=90^{\circ}\right)$ than that of $\theta_{1}\left(\approx 7^{\circ}\right.$ for $\left.i=90^{\circ}\right)$, as shown recently by Laughlin et al. (2004).

Lee \& Peale (2002) have shown that if sufficient eccentricity damping $\left(\dot{e}_{2} / e_{2} \approx 100 \dot{a}_{2} / a_{2}\right)$ is applied during migration, the observed configuration of GJ 876 can be maintained for arbitrary migration times. We have updated the amount of eccentricity damping required using the updated dynamical fits by Laughlin et al. (2004). For the best fits with coplanar inclination $i \gtrsim 35^{\circ}, e_{1} \lesssim 0.31, e_{2} \lesssim 0.05$, and $\dot{e}_{2} / e_{2}$ must exceed $\approx 40 \dot{a}_{2} / a_{2}$. However, no such rapid eccentricity damping mechanism is presently known. The hydrodynamic simulations typically give comparable time scales for eccentricity damping and semi-major axis decrease. Some current theories of planetdisk interactions indicate that eccentricity driving should occur (Ogilvie \& Lubow 2003; Goldreich \& Sari 2003). Such eccentricity behavior would not be consistent with the observed state of GJ 876 if there were extensive migration after capture into the resonances.

If the disk is removed soon after the planets are captured into resonance, as in Figs. 13 to 15, the driving disappears and the final eccentricities are smaller, like those observed. However, the disk must vanish before the post-capture orbits shrink by $\sim 10 \%$, similar to the result found in 3-body integrations by Lee \& Peale (2002). We have used in the present simulations a high viscosity and high surface density to increase the migration rates, and to be able to perform the simulations in a reasonable amount of computer time. In turn this implies a need for rapid disk dispersal to halt migration. If more realistic values were used for the viscosity and the disk mass, the necessary disk dispersal time scale could be much more extended. What appears to be somewhat fine tuned in the present simulations would be perhaps more reasonable. Our scenario only requires that capture occurred in the final formation phase of GJ 876, and that the planets did not migrate over a very long distance while locked in the resonances.
Additional effects that might influence the eccentricity damping in these type of simulations are possibly threedimensional. Fully 3D-simulations of circular planets on a fixed orbit (D'Angelo et al. 2003b; Bate et al. 2003) have shown that the spiral structures are not so clear and material may enter the Roche lobe from above the midplane, an effect which may alter the eccentricity damping properties. The inclusion of magnetic fields might lead to a magnetic coupling of the planetary field with that of the disk, which will have an influence on the eccentricity evolution. But if the consideration of these and perhaps other processes fail to produce sufficient eccentricity damping, the elimination of the disk shortly after the capture of the GJ 876 planets into the $2: 1$ resonances is a possible, albeit perhaps unsatisfying means of accounting for the observed low-eccentricity configuration.

Acknowledgements. We would like to thank Greg Laughlin for stimulating discussions during the course of this project and for furnishing the details of the dynamical fits by Laughlin et al. (2004). We also thank D. N. C. Lin and M. Nagasawa for informative discussions. The work was sponsored by the KITP Program "Planet Formation: Terrestrial and Extra-Solar" held in Santa Barbara from January 2004 until March 2004. We thank the organizers for providing a pleasant and very stimulating atmosphere. This research was supported in part by the National Science Foundation under Grant No. PHY99-0794 and by NASA grants NAG5-11666 and NAG5-13149.

\section{References}

Bate, M. R., Lubow, S. H., Ogilvie, G. I., \& Miller, K. A. 2003, MNRAS, 341, 213

Beaugé, C., Ferraz-Mello, S., \& Michtchenko, T. A. 2003, ApJ, 593, 1124

Beaugé, C., \& Michtchenko, T. A. 2003, MNRAS, 341, 760

Benedict, G. F., McArthur, B. E., Forveille, T., et al. 2002, ApJ, 581, L115

Bryden, G., Chen, X., Lin, D. N. C., Nelson, R. P., \& Papaloizou, J. C. B. 1999, ApJ, 514, 344

Bryden, G., Różyczka, M., Lin, D. N. C., \& Bodenheimer, P. 2000, ApJ, 540, 1091

D’Angelo, G., Bate, M. R., \& Lubow, S. H. 2004, [arXiv: astro-ph/0411705], MNRAS, in press

D’Angelo, G., Henning, T., \& Kley, W. 2002, A\&A, 385, 647

D’Angelo, G., Henning, T., \& Kley, W. 2003a, ApJ, 599, 548

D'Angelo, G., Kley, W., \& Henning, T. 2003b, ApJ, 586, 540

Delfosse, X., Forveille, T., Mayor, M., et al. 1998, A\&A, 338, L67

Duncan, M. J., Levison, H. F., \& Lee, M. H. 1998, AJ, 116, 2067

Ferraz-Mello, S., Beaugé, C., \& Michtchenko, T. A. 2003, Celest. Mech. Dynam. Astron., 87, 99

Günther, R., Schäfer, C., \& Kley, W. 2004, A\&A, 423, 559

Goldreich, P., \& Sari, R. 2003, ApJ, 585, 1024

Hubeny, I. 1990, ApJ, 351, 632

Kley, W. 1989, A\&A, 208, 98

Kley, W. 1998, A\&A, 338, L37

Kley, W. 1999, MNRAS, 303, 696

Kley, W. 2000, MNRAS, 313, L47

Kley, W., Peitz, J., \& Bryden, G. 2004, A\&A, 414, 735

Laughlin, G., Butler, R. P., Fischer, D. A., et al. 2004, [arXiv: astro-ph/0407441], ApJ, in press

Laughlin, G., \& Chambers, J. E. 2001, ApJ, 551, L109

Lee, M. H. 2004, ApJ, 611, 517

Lee, M. H., \& Peale, S. J. 2002, ApJ, 567, 596 
Lin, D. N. C., \& Papaloizou, J. C. B. 1985, in Protostars and Planets II, 981

Lubow, S. H. 1991, ApJ, 381, 259

Marcy, G. W., Butler, R. P., Fischer, D. A., et al. 2002, ApJ, 581, 1375

Marcy, G. W., Butler, R. P., Fischer, D. A., et al. 2001, ApJ, 556, 296

Marcy, G. W., Butler, R. P., Vogt, S. S., Fischer, D. A., \& Lissauer, J. J. 1998, ApJ, 505, L147

Mayor, M., Udry, S., Naef, D., et al. 2004, A\&A, 415, 391

McArthur, B. E., Endl, M., Cochran, W. D., et al. 2004, ApJ, 614, L81

Mihalas, D., \& Weibel Mihalas, B. 1984, Foundations of radiation hydrodynamics (New York: Oxford University Press)

Murray, N., Paskowitz, M., \& Holman, M. 2002, ApJ, 565, 608

Nelson, R. P., \& Papaloizou, J. C. B. 2002, MNRAS, 333, L26
Nelson, R. P., Papaloizou, J. C. B., Masset, F. S., \& Kley, W. 2000, MNRAS, 318, 18

Ogilvie, G. I., \& Lubow, S. H. 2003, ApJ, 587, 398

Papaloizou, J. C. B. 2003, Celest. Mech. Dynam. Astron., 87, 53

Papaloizou, J. C. B., Nelson, R. P., \& Masset, F. 2001, A\&A, 366, 263

Rivera, E. J., \& Lissauer, J. J. 2001, ApJ, 558, 392

Snellgrove, M. D., Papaloizou, J. C. B., \& Nelson, R. P. 2001, A\&A, 374, 1092

van Leer, B. 1977, J. Comput. Phys., 23, 276

Varnière, P., Quillen, A. C., \& Frank, A. 2004, ApJ, 612, 1152

Ward, W. R. 1981, Icarus, 47, 234

Ward, W. R. 1997, Icarus, 126, 261

Yoder, C. F., \& Peale, S. J. 1981, Icarus, 47, 1 